\title{
Harnack inequality for hypoelliptic ultraparabolic equations with a singular lower order term
}

\section{Sergio Polidoro and Maria Alessandra Ragusa}

\begin{abstract}
We prove a Harnack inequality for the positive solutions of ultraparabolic equations of the type

$$
\mathcal{L}_{0} u+\mathcal{V} u=0,
$$

where $\mathcal{L}_{0}$ is a linear second order hypoelliptic operator and $\mathcal{V}$ belongs to a class of functions of Stummel-Kato type. We also obtain the existence of a Green function and an uniqueness result for the CauchyDirichlet problem.
\end{abstract}

\section{Introduction}

We prove some regularity results for the solutions of the equation in $\mathbb{R}^{N+1}$

$$
\mathcal{L}_{0} u+\mathcal{V} u=0
$$

where $\mathcal{V}$ is a singular potential belonging to a Stummel-Kato class (see Definition 1.1 below) and $\mathcal{L}_{0}$ is a linear second order operator of the form

$$
\mathcal{L}_{0}=\sum_{k=1}^{m} X_{k}^{2}+X_{0}-\partial_{t} .
$$

We always denote by $z=(x, t)$ the point in $\mathbb{R}^{N+1}$; the $X_{k}$ 's in $(1.2)$ are smooth vector fields on $\mathbb{R}^{N}$, i.e.

$$
X_{k}(x)=\sum_{j=1}^{N} a_{j}^{k}(x) \partial_{x_{j}}, \quad k=0, \ldots, m,
$$

2000 Mathematics Subject Classification: 35K70, 35J10, 35K20, 32A37, 35B65.

Keywords: Hypoelliptic operator, Schrödinger equation, Harnack inequality, Green function. 
where any $a_{j}^{k}$ is a $C^{\infty}$ function. In the sequel we also consider the $X_{k}$ 's as vector fields in $\mathbb{R}^{N+1}$ and we denote

$$
Y=X_{0}-\partial_{t}
$$

We also assume that $X_{0}(0)=0$, so that

$$
Y(0)=-\partial_{t}
$$

As we will see in Remark 3.2, this assumption is not necessary, but allows us to simplify the notations.

We say that a curve $\gamma:[0, T] \rightarrow \mathbb{R}^{N+1}$ is L-admissible if it is absolutely continuous and satisfies

$$
\gamma^{\prime}(s)=\sum_{k=1}^{m} \lambda_{k}(s) X_{k}(\gamma(s))+\mu(s) Y(\gamma(s)), \quad \text { a.e. in }[0, T],
$$

for suitable piecewise constant real functions $\lambda_{1}, \ldots, \lambda_{m}, \mu$, with $\mu \geq 0$. We next state our main assumptions:

[H.1] there exists a homogeneous Lie group $\mathbb{G}=\left(\mathbb{R}^{N+1}, \circ, \delta_{\lambda}\right)$ such that

(i) $X_{1}, \ldots, X_{m}, Y$ are left translation invariant on $\mathbb{G}$;

(ii) $X_{1}, \ldots, X_{m}$ are $\delta_{\lambda}$-homogeneous of degree one and $Y$ is $\delta_{\lambda}$-homogeneous of degree two;

[H.2] for every $(x, t),(\xi, \tau) \in \mathbb{R}^{N+1}$ with $t>\tau$, there exists an $L$-admissible path $\gamma:[0, T] \rightarrow \mathbb{R}^{N+1}$ such that $\gamma(0)=(x, t), \gamma(T)=(\xi, \tau)$.

Operators of this kind have been studied by Kogoj and Lanconelli in [11]. The above hypotheses and the main properties of homogeneous Lie groups will be discussed in detail in the next section, here we recall that assumptions [H.1]-[H.2] yield the well known Hörmander condition [10]:

$$
\operatorname{rank} \operatorname{Lie}\left\{X_{1}, \ldots, X_{m}, Y\right\}(z)=N+1, \quad \text { for every } z \in \mathbb{R}^{N+1},
$$

then $\mathcal{L}_{0}$ is hypoelliptic (i.e. every distributional solution to $\mathcal{L}_{0} u=f$ is smooth whenever $f$ is smooth; see, for instance, Proposition 10.1 in [11]). Hence $\mathcal{L}_{0}$ belongs to the general class of the hypoelliptic operators on homogeneous groups first studied by Folland [8]. We recall that a general theory of function spaces related to Hörmander operators has been developed by Rothschild and Stein in [24], and by Nagel, Stein and Wainger in [21]. An invariant Harnack inequality for the positive solutions of $\mathcal{L}_{0} u=0$ and a Gaussian upper estimate of its fundamental solution $\Gamma_{0}$ have been proved 
in [11]. We also recall that Gaussian lower bounds for operators verifying assumptions [H.1]-[H.2] on Lie group of step three have been given in [22]; and one-side Liouville theorems are provided in [12].

Let us point out that several meaningful examples of operators of the form (1.2) satisfy assumptions [H.1]-[H.2]:

- heat operators on Carnot groups

$$
\Delta_{\mathbb{G}}-\partial_{t},
$$

where $\Delta_{\mathbb{G}}=\sum_{k=1}^{m} X_{k}^{2}$ denotes the sub-Laplacian on a homogeneous Carnot group $\mathbb{G}$ (see Varopoulos, Saloff-Coste and Coulhon [28]);

- heat operators with drift on Carnot groups

$$
\Delta_{\mathbb{G}}+X_{0}-\partial_{t}
$$

(see Alexopoulos [1]). Note that, due to our assumption [H.1], the vector field $X_{0}$ belongs to the second layer of the Lie algebra of the group);

- Kolmogorov type operators

$$
\Delta_{\mathbb{R}^{m}}+\langle B x, \nabla\rangle-\partial_{t},
$$

where $\Delta_{\mathbb{R}^{m}}$ is the Laplace operator on $\mathbb{R}^{m}$ and $B$ is a constant $N \times N$ real matrix (see [16] and its bibliography for a survey on known results on Kolmogorov type operators. In [18] necessary and sufficient conditions are given on the matrix $B$ in order to satisfy assumptions [H.1]-[H.2]);

- operators on the "link of a Carnot and a Kolmogorov group"

$$
\Delta_{\mathbb{G}}+\langle B x, \nabla\rangle-\partial_{t}
$$

here the domain of the solution is $\mathbb{R}^{m} \times \mathbb{R}^{p} \times \mathbb{R}^{q} \times \mathbb{R}, \mathbb{G}$ is a Carnot group on $\mathbb{R}^{m} \times \mathbb{R}^{p}$ and $B$ is a $(m+q) \times(m+q)$ matrix as in the Kolmogorov operator (1.8) (see Kogoj and Lanconelli [11, Example 9.7]). A general procedure for the construction of sequences of linked groups of dimension and step arbitrarily large is given in the paper [13].

We are concerned with the regularity of the operator

$$
\mathcal{L}_{\mathcal{V}}=\mathcal{L}_{0}+\mathcal{V}
$$

where $\mathcal{V}$ belongs to the following Stummel-Kato class (defined by the fundamental solution $\Gamma_{0}$ of $\mathcal{L}_{0}$ ). 
Definition 1.1. Let $\Omega$ be an open subset contained in $\mathbb{R}^{N+1}$. A function $\mathcal{V} \in L^{1}(\Omega)$ belongs to the space $S K\left(\Omega, \mathcal{L}_{0}\right)$, if

$$
\lim _{h \rightarrow 0} \eta_{\mathcal{V}}(h)=0, \quad \lim _{h \rightarrow 0} \eta_{\mathcal{V}}^{*}(h)=0,
$$

where

$$
\begin{aligned}
& \eta_{\mathcal{V}}(h)=\sup _{(x, t) \in \Omega} \int_{(y, s) \in \Omega,} \underset{t-h^{2}<s<t}{\Gamma_{0}(x, t, y, s)|\mathcal{V}(y, s)| d y d s,} \\
& \eta_{\mathcal{V}}^{*}(h)=\sup _{(y, s) \in \Omega} \int_{(x, t) \in \Omega, s<t<s+h^{2}} \Gamma_{0}(x, t, y, s)|\mathcal{V}(x, t)| d x d t .
\end{aligned}
$$

We say that $u$ is a weak solution of $\mathcal{L}_{\mathcal{V}} u=0$ if

1. there exists $p>1$ such that $u, X_{1} u, \ldots, X_{m} u \in L_{\mathrm{loc}}^{p}(\Omega)$,

2. $\mathcal{V} u \in L_{\text {loc }}^{1}(\Omega)$,

3. $\int_{\Omega} \sum_{k=1}^{m} X_{k} u X_{k}^{*} \varphi+\int_{\Omega} u Y^{*} \varphi+\int_{\Omega} u \mathcal{V} \varphi=0$, for every $\varphi \in C_{0}^{\infty}(\Omega)$.

As in the Euclidean setting, the Stummel-Kato class can be related to the Morrey spaces $L^{p, \lambda}(\Omega, \mathbb{G})$; in Section 3 we will prove the inclusion $L^{1, \lambda}(\Omega, \mathbb{G}) \subset S K\left(\Omega, \mathcal{L}_{0}\right)$ for $\left.\lambda \in\right] Q-2, Q[$, where $Q$ is the homogeneous dimension of $\mathbb{G}$ (see Section 2 for the definitions). We also give a simple sufficient condition for the integrability of $\mathcal{V} u$ : we show that, if the derivatives $X_{j} u, X_{j} X_{k} u$, for $j, k=1, \ldots, m$ and $Y u$ belong to $L_{\text {loc }}^{1}(\Omega)$ then $\mathcal{V} u \in L_{\text {loc }}^{1}(\Omega)$.

Our main result is an invariant Harnack inequality for the positive solutions to $\mathcal{L}_{\mathcal{V}} u=0$. The proof of the Harnack inequality given by Kogoj and Lanconelli in [11] (for the solutions to $\mathcal{L}_{0} u=0$ ) is based on a mean value theorem and follows the same lines of the classical proof of the Harnack inequality for harmonic functions. That approach has been used in the study of Kolmogorov operators (1.8) by Kuptsov in [14], later by Garofalo and Lanconelli in [9] then by Lanconelli and Polidoro in [18] and relies on some accurate estimates of the derivatives $X_{1} \Gamma_{0}, \ldots X_{m} \Gamma_{0}$ of the fundamental solution of $\mathcal{L}_{0}$. Here we use a method based on the Green function $G_{0}$ of $\mathcal{L}_{0}$ related to suitable "cylindrical" open sets and on a pointwise lower bound for $G_{0}$. This technique is inspired by some arguments by Safanov in [25], and used in [15] where Kusuoka and Stroock obtain Harnack inequality for solutions to certain degenerate equations. It has been also used by Fabes and Stroock in [6], [7] to study uniformly elliptic and parabolic operators with measurable coefficients and later adapted by Montanari in [20] to obtain a Harnack inequality for $\mathcal{L}_{0}$ belonging to a class of totally degenerate hypoelliptic operators. The same method has been successfully used by the authors in [23], in the study of Kolmogorov operators (1.8). 
We finally recall some papers where the second order part of the operator $\mathcal{L}_{0}$ has non-smooth coefficients. We quote Sturm [27] and Zhang [29], that consider the operator (1.10) where $\mathcal{L}_{0}$ is uniformly parabolic, Citti, Garofalo and Lanconelli [5] and Lu [19], who consider the Schrödinger operator related to sum of square of Hörmander's vector fields $\mathcal{L}_{\mathcal{V}}=\sum_{k=1}^{m} X_{k}^{2}+\mathcal{V}$, Zhang [30], who studies the analogous parabolic operator $\mathcal{L}_{\mathcal{V}}=\sum_{k=1}^{m} X_{k}^{2}-$ $\partial_{t}+\mathcal{V}$ as (1.6). Recently Bramanti and Brandolini in [4] consider operators, without potential function, of the following type: $\mathcal{L}=\sum_{i, j=1}^{m} a_{i j}(x) X_{i} X_{j}$, where $a_{i j}$ belong to the the Sarason class $V M O$. They extend to spaces of homogeneous type some regularity estimates.

We end this introduction with a short outline of this paper. In Section 2 we recall the known facts about homogeneous Lie groups and on the boundary value problems for $\mathcal{L}_{0}$, that will be needed through in the sequel, then we state our main results. In Section 3 we discuss the main properties of the fundamental solution and of the Green function for $\mathcal{L}_{0}$. In Section 4 we construct a Green function for $\mathcal{L}_{\mathcal{V}}$ by the Levi parametrix method; some $L^{p}$ estimates and a pointwise lower bound for the Green function are proved. Then, in Section 5 we prove the results of this paper, in a preliminary statement only for bounded potentials $\mathcal{V}$, then, by a limiting argument, for every $\mathcal{V}$ in the Stummel-Kato class.

\section{Known facts and statement of main results}

In this section we briefly recall the basic properties of the homogeneous Lie groups and the related homogeneous vector fields. We refer to the recent monograph [2] by Bonfiglioli, Lanconelli and Uguzzoni, for a more exhaustive treatment of that topic. We state our main results in the last part of the section.

A Lie group $\mathbb{G}=\left(\mathbb{R}^{N+1}, \circ\right)$ is said homogeneous if there exists a family of dilations $\left(\delta_{\lambda}\right)_{\lambda>0}$ of the form

$$
\delta_{\lambda}: \mathbb{R}^{N+1} \rightarrow \mathbb{R}^{N+1}, \quad \delta_{\lambda}\left(x_{1}, \ldots, \xi_{N}, t\right)=\left(\lambda^{\alpha_{1}} x_{1}, \ldots, \lambda^{\alpha_{N}} \xi_{N}, \lambda^{\alpha_{0}} t\right)
$$

for some positive $\alpha_{1}, \ldots, \alpha_{N}, \alpha_{0}$, with the following property

$$
\delta_{\lambda}(z \circ \zeta)=\left(\delta_{\lambda} z\right) \circ\left(\delta_{\lambda} \zeta\right), \quad \text { for every } z, \zeta \in \mathbb{R}^{N+1} \text { and } \lambda>0 \text {. }
$$

Hypotheses [H.1]-[H.2] imply that $\mathbb{R}^{N}$ has a direct sum decomposition

$$
\mathbb{R}^{N}=V_{1} \oplus \cdots \oplus V_{n}
$$

such that, if we decompose any point $x \in \mathbb{R}^{N}$ as $x=x^{(1)}+\cdots+x^{(n)}$ with $x^{(k)} \in V_{k}$, then the dilations are

$$
\delta_{\lambda}\left(x^{(1)}+\cdots+x^{(n)}, t\right)=\left(\lambda x^{(1)}+\cdots+\lambda^{n} x^{(n)}, \lambda^{2} t\right),
$$


for any $\lambda>0$. If we let $m_{k}=\operatorname{dim} V_{k}$, the natural number

$$
Q=2+\sum_{k=1}^{n} k m_{k}
$$

is usually called the homogeneous dimension of $\mathbb{G}$ with respect to $\left(\delta_{\lambda}\right)_{\lambda>0}$. We also introduce the following $\delta_{\lambda}$-homogeneous norms on $\mathbb{R}^{N+1}$ and $\mathbb{R}^{N}$ :

$$
\|(x, t)\|_{\mathbb{G}}=\left(\sum_{k=1}^{n}\left|x^{(k)}\right|^{\frac{2 n !}{k}}+|t|^{n !}\right)^{\frac{1}{2 n !}} \quad|x|_{\mathbb{G}}=\left(\sum_{k=1}^{n}\left|x^{(k)}\right|^{\frac{2 n !}{k}}\right)^{\frac{1}{2 n !}}
$$

$\left(\left|x^{(k)}\right|\right.$ is the Euclidean norm of $\left.x^{(k)}\right)$. We denote by

$$
d(z, \zeta)=\left\|\zeta^{-1} \circ z\right\|_{\mathbb{G}}
$$

the quasi-distance between two points $z, \zeta \in \mathbb{R}^{N+1}$, and by

$$
B_{r}(z)=\left\{\zeta \in \mathbb{R}^{N+1}: d(z, \zeta)<r\right\}
$$

the ball with center at $z$ and radius $r$. Recall that there exists a positive constant $c$ such that

$$
d(z, w) \leq c(d(z, \zeta)+d(\zeta, w)), \quad d(z, w) \leq c d(w, z),
$$

for every $z, \zeta, w \in \mathbb{R}^{N+1}$ (see [8], Proposition 1.4).

We also recall that, due to the fact that $X_{0}, \ldots, X_{m}$ only depend on the space variable $x$, the composition law $\circ$ is Euclidean in the time variable $t$, i.e.

$$
(x, t) \circ(y, s)=(\sigma(x, t, y, s), t+s)
$$

for a suitable smooth function $\sigma$ (see [11], Proposition 10.2). Moreover, since $X_{1}, \ldots, X_{m}$ and $Y$ are homogeneous vector fields of degree 1 and 2, respectively, we have

$$
\begin{aligned}
& ((x, t) \circ(y, s))^{(1)}=x^{(1)}+y^{(1)}, \\
& ((x, t) \circ(y, s))^{(k)}=x^{(k)}+y^{(k)}+\sigma_{k}(x, t, y, s)
\end{aligned}
$$

for $k=2, \ldots, m$, where $\sigma_{k}(x, t, y, s)$ is a polynomial function that only depends on $x^{(k+1)}+\cdots+x^{(m)}, t, y^{(k+1)}+\cdots+y^{(m)}$ and $s$. As a consequence, the determinant of the Jacobian matrix of the function $z \mapsto z_{0} \circ z$ equals one, thus the Lebesgue measure of $\mathbb{R}^{N+1}$ is left-invariant under left translations, namely

$$
\operatorname{meas}\left(z_{0} \circ E\right)=\operatorname{meas}(E),
$$

for every $z_{0} \in \mathbb{R}^{N+1}$ and every measurable set $E \subset \mathbb{R}^{N+1}$. 
Another consequence of the homogeneity of the vector fields $X_{1}, \ldots, X_{m}$ and $Y$ is that they are of the form

$$
\begin{aligned}
X_{k} & =\sum_{j=1}^{n} a_{j-1}^{k}\left(x^{(1)}, \ldots, x^{(j-1)}\right) \cdot \nabla^{(j)}, \quad k=1, \ldots, m, \\
Y & =\sum_{j=2}^{n} b_{j-2}\left(x^{(1)}, \ldots, x^{(j-2)}\right) \cdot \nabla^{(j)}-\partial_{t},
\end{aligned}
$$

where $\nabla^{(j)}=\left(0, \ldots, 0, \partial_{x_{1}^{(j)}}, \ldots, \partial_{x_{m_{j}}^{(j)}}, 0, \ldots, 0\right)$ denotes the gradient with respect to the variable $x^{(j)}$ and $a_{j}^{k}$ and $b_{j}$ are $\delta_{\lambda}$-homogeneous polynomial functions of degree $j$ with values in $V_{j+1}$ and $V_{j+2}$ respectively. As a first consequence we have that $X_{k}^{*}=-X_{k}$ for $k=1, \ldots, m$ and $Y^{*}=-Y$, thus the formal adjoint of $\mathcal{L}_{0}$ is $\mathcal{L}_{0}{ }^{*}=\sum_{k=1}^{m} X_{k}^{2}-Y$.

Let us explicitly note that hypothesis [H.2] and formula (2.7) imply that, if we write $\mathcal{L}_{0}$ as

$$
\mathcal{L}_{0} \equiv \sum_{i, j=1}^{N} a_{i, j}(x) \partial_{x_{i} x_{j}}+\sum_{j=1}^{N} b_{j}(x) \partial_{x_{j}}-\partial_{t},
$$

then the $m \times m$ block matrix $\left(a_{i, j}(x)\right)_{i, j=1, \ldots, m}$ is constant and positive definite.

We next recall some results, due to Lanconelli and Pascucci [17], concerning the boundary value problem for $\mathcal{L}_{0}$. Let $k \in \mathbb{N}$ and $\varepsilon>0$ be two constants that will be chosen in the sequel. We denote

$$
O=B_{\operatorname{eucl}\left(k e_{1}, k+k \varepsilon\right)} \cap B_{\text {eucl }\left(-k e_{1}, k+k \varepsilon\right)},
$$

where $B_{\text {eucl }(x, r)}$ is the Euclidean ball of $\mathbb{R}^{N}$ with center at $x$ and radius $r$. Moreover, for positive $T$ we let

$Q(T)=O \times] 0, T[, S=O \times\{0\}, S(T)=O \times\{T\}$, and $M(T)=\partial O \times] 0, T[$

be the "unit" cylinder of $\mathbb{R}^{N+1}$, its lower and upper basis (resp.), and its lateral boundary. We will call parabolic boundary of $Q(T)$ the set

$$
\partial_{r} Q(T)=S \cup(\partial O \times[0, T]) .
$$

Finally, for every positive $R$ and for any $(\xi, \tau) \in \mathbb{R}^{N+1}$, we set

$$
Q_{R}(\xi, \tau, T)=(\xi, \tau) \circ \delta_{R}\left(Q\left(R^{-2} T\right)\right),
$$


and, analogously,

$$
\begin{aligned}
& M_{R}(\xi, \tau, T)=(\xi, \tau) \circ \delta_{R}\left(M\left(R^{-2} T\right)\right), S_{R}(\xi, \tau)=(\xi, \tau) \circ \delta_{R}(S), \\
& S_{R}(\xi, \tau, T)=(\xi, \tau) \circ \delta_{R}\left(S\left(R^{-2} T\right)\right), \partial_{r} Q_{R}(\xi, \tau, T)=(\xi, \tau) \circ \delta_{R}\left(\partial_{r} Q\left(R^{-2} T\right)\right)
\end{aligned}
$$

(note that, by (2.2) and (2.4), $T$ is the true height of the sets $Q_{R}(\xi, \tau, T)$, $M_{R}(\xi, \tau, T)$ and $\partial_{r} Q_{R}(\xi, \tau, T)$, and $S_{R}(\xi, \tau, T)=Q_{R}(\xi, \tau, T) \cap\{(x, t): t=$ $\tau+T\})$. We also remark that, by (2.2) and (2.6), we have

$$
\text { meas }\left(Q_{R}\left(\xi, \tau, R^{2} T\right)\right)=R^{Q} \text { meas }(Q(T)) \text {. }
$$

Moreover

$$
\operatorname{meas}\left(S_{R}(\xi, \tau)\right)=R^{Q-2} \operatorname{meas}(S),
$$

where, with a slight abuse of notations, meas $\left(S_{R}(\xi, \tau)\right)$ is the $N$-dimensional measure of the set $S_{R}(\xi, \tau)$ and, obviously,

$$
\text { meas }\left(Q_{R}\left(\xi, \tau, R^{2} T\right)\right)=T R^{Q} \text { meas }(S) \text {. }
$$

Consider the Cauchy-Dirichlet problem in the unit cylinder

$$
\begin{cases}\mathcal{L}_{0} u=f & \text { in } Q(T) \\ u=0 & \text { in } \partial_{r} Q(T)\end{cases}
$$

with $f \in C_{0}^{\infty}(Q(T))$. As noticed before, the $m \times m$ block matrix $\left(a_{i, j}(x)\right)_{i, j=1, \ldots, m}$ in (2.8) is constant and positive definite so that, in particular, $a_{11}>0$. Then, by Proposition 2.4 and Theorem 2.5 in $[17]^{1}$ there exists a positive $\varepsilon$ in the definition of $O$ such that the Dirichlet problem (2.12) has a unique (classical) solution $u \in C\left(Q(T) \cup \partial_{r} Q(T)\right) \cap C^{\infty}(Q(T))$ (in the sequel $\varepsilon$ in the definition of $O$ will be always chosen as above).

We say that $G_{0}:\left(Q(T) \cup \partial_{r} Q(T)\right) \times Q(T) \rightarrow \mathbb{R}$ is a Green function for $Q(T)$ if, for every $f \in C_{0}\left(S_{R}\right)$, the function

$$
u(z)=-\int_{Q(T)} G_{0}(z, \zeta) f(\zeta) d \zeta
$$

is solution of the Cauchy-Dirichlet problem (2.12). In [17], Theorem 2.7 it is proved that a Green function $G_{0}$ exists and is smooth out of the diagonal of the set $\left(Q(T) \cup \partial_{r} Q(T)\right) \times Q(T) ; G_{0}(x, t, \xi, \tau) \geq 0$; for any $(x, t),(\xi, \tau) \in$ $\mathbb{R}^{N+1}, G_{0}(x, t, \xi, \tau)=0$ if, $t \leq \tau$.

\footnotetext{
${ }^{1}$ in [17] it is assumed that $\mathcal{L}_{0}$ is the heat operator out of a compact set of $\mathbb{R}^{N+1}$. $\mathcal{L}_{0}$ can be suitably modified outside $Q(T)$ in order to fulfill such a requirement.
} 
The function $G_{0}^{*}(z, \zeta) \equiv G_{0}(\zeta, z)$ is a Green function for the adjoint operator $\mathcal{L}_{0}{ }^{*}$. For every positive $R$ and for any $(\xi, \tau) \in \mathbb{R}^{N+1}$ the function $G_{0}\left((\xi, \tau) \circ \delta_{R}(\zeta),(\xi, \tau) \circ \delta_{R}(z)\right)$ is a Green function for the set $Q_{R}(\xi, \tau, T)$. The Green function can be characterized as

$$
G_{0}(x, t, y, \tau)=\Gamma_{0}(x, t, y, \tau)-h(x, t, y, \tau)
$$

where $h(\cdot, \cdot, y, 0)$ is the solution of the boundary value problem

$$
\begin{cases}\mathcal{L}_{0} u=0 & \text { in } Q(T) \\ u=0 & \text { in } M(T) \\ u=\Gamma_{0}(\cdot, \cdot, y, 0) & \text { in } S\end{cases}
$$

The existence of a generalized solution $h$ can be proved by a standard method, based on the elliptic regularization procedure. An universal barrier at every point of $M(T) \cup S$ has been constructed in the proof of Theorem 2.5 in [17], then $h$ attains the boundary data by continuity. Moreover, by the hypoellipticity of $\mathcal{L}_{0}, h$ is a smooth classical solution to $\mathcal{L}_{0} u=0$ in $Q(T)$. Since $G_{0}(\zeta, \cdot)$ is a Green function for the adjoint operator $\mathcal{L}_{0}{ }^{*}$, we also have that $h$ is smooth for $(x, t) \neq(y, 0)$. By the minimum principle it plainly follows $h \geq 0$, then

$$
G_{0}(x, t, y, s) \leq \Gamma_{0}(x, t, y, s), \quad \text { for every }(x, t),(y, s) \in \mathbb{R}^{N+1} .
$$

We finally note that, for any $\varphi \in C_{0}\left(\mathbb{R}^{N}\right)$, the function

$$
u(x, t)=\int_{\mathbb{R}^{N}} \Gamma_{0}(x, t, y, 0) \varphi(y) d y
$$

is a classical solution to the Cauchy problem $\mathcal{L}_{0} u=0$ in $\mathbb{R}^{N} \times \mathbb{R}^{+}, u(x, 0)=$ $\varphi(x)$; as a consequence, for every $\varphi \in C_{0}(S)$, the function

$$
v(x, t)=\int_{S} G_{0}(x, t, y, 0) \varphi(y) d y
$$

is a classical solution to the Cauchy-Dirichlet problem $\mathcal{L}_{0} u=0$ in $Q(T)$, $u=\varphi$ in $S$ and $u \equiv 0$ in $M(T)$.

We next state the main results of this note. For every $R, T>0$ and $(\xi, \tau) \in \mathbb{R}^{N+1}$, consider the Cauchy-Dirichlet problem

$$
\begin{cases}\mathcal{L}_{\mathcal{V}} u=f & \text { in } Q_{R}(\xi, \tau, T) \\ u=0 & \text { in } \partial_{r} Q_{R}(\xi, \tau, T)\end{cases}
$$

with $f \in C_{0}\left(Q_{R}(\xi, \tau, T)\right)$. 
We say that $u$ is a weak solution of (2.16) if it is a weak solution to $\mathcal{L}_{\mathcal{V}} u=f$ in $Q_{R}(\xi, \tau, T)$, it belongs to $C\left(Q_{R}(\xi, \tau, T) \cup \partial_{r} Q_{R}(\xi, \tau, T)\right)$ and attains the boundary data by continuity. We say that

$$
G:\left(Q_{R}(\xi, \tau, T) \cup \partial_{r} Q_{R}(\xi, \tau, T)\right) \times Q_{R}(\xi, \tau, T) \rightarrow \mathbb{R}
$$

is a Green function for $(2.16)$ if $G(\cdot, w)$ is a weak solution to $(2.16)$, for every $w \in Q_{R}(\xi, \tau, T)$.

Theorem 2.1. The Cauchy-Dirichlet problem (2.16) has a unique weak solution $u$. Moreover a Green function $G$ for $Q_{R}(\xi, \tau, T)$ exists, and the function $G^{*}(\zeta, z)=G(z, \zeta)$ is a Green function for the adjoint operator $\mathcal{L}_{\mathcal{V}}{ }^{*}$.

Before stating our second result, we introduce two further notations. Let us consider the cylinder $Q_{R}\left(\xi, \tau, R^{2}\right)$ and, for every $\left.\alpha, \beta, \gamma, \delta \in\right] 0,1[: \alpha<\beta<\gamma$, let us set

$$
\begin{aligned}
& Q^{-}=\left\{(x, t) \in Q_{\delta R}\left(\xi, \tau, R^{2}\right): \tau+\alpha R^{2} \leq t \leq \tau+\beta R^{2}\right\} \\
& Q^{+}=\left\{(x, t) \in Q_{\delta R}\left(\xi, \tau, R^{2}\right): \tau+\gamma R^{2} \leq t\right\} .
\end{aligned}
$$

Theorem 2.2. (Harnack). Let $\mathcal{V} \in S K\left(\Omega, \mathcal{L}_{0}\right)$. Then there exist two constants $R_{0}>0$ and $\left.\delta_{0} \in\right] 0,1\left[\right.$ such that, for every $Q_{R}\left(\xi, \tau, R^{2}\right) \subset \subset \Omega$, with $R \leq R_{0}$ and $Q^{+}, Q^{-}$as above, with $\left.\delta \in\right] 0, \delta_{0}[$, we have

$$
\sup _{Q^{-}} u \leq M \inf _{Q^{+}} u
$$

for every positive weak solution $u$ of $\mathcal{L}_{\mathcal{V}} u=0$. Here $M$ is a positive constant that depends on $\eta_{\mathcal{V}}, \eta_{\mathcal{V}}^{*}$ and on the constants $\alpha, \beta, \gamma, \delta$.

Proposition 2.3. If $u$ is a weak solution of $\mathcal{L}_{\mathcal{V}} u=0$ in $\Omega$, with $\mathcal{V} \in$ $\operatorname{SK}\left(\Omega, \mathcal{L}_{0}\right)$, then $u$ is continuous. Moreover, for any $\left.\beta \in\right] 0,1[$ there exists a positive constant $C_{\beta}$, dependent only on $\mathcal{L}_{0}$ and $\beta$, such that

$$
\left|u(z)-u\left(z_{0}\right)\right| \leq\left(C_{\beta} d\left(z, z_{0}\right)^{\beta}+2 \eta_{\mathcal{V}}\left(5 c^{2} d\left(z, z_{0}\right)^{1-\beta}\right)\right) \sup _{B_{4 r}\left(z_{0}\right)}|u|
$$

for every $\left.z_{0} \in \Omega, r \in\right] 0,1\left[\right.$ such that $B_{4 r}\left(z_{0}\right) \subset \Omega$ and for every $z \in B_{r_{\beta}}\left(z_{0}\right)$ (where $r_{\beta}=r^{\frac{1}{1-\beta}}$ and $c$ is the constant in (2.3)).

If $\mathcal{V} \in L^{1, \lambda}(\Omega, \mathbb{G})$ with $\left.\lambda \in\right] Q-2, Q[$, then

$$
\left|u(z)-u\left(z_{0}\right)\right| \leq C_{\beta}\left(1+\|\mathcal{V}\|_{L^{1, \lambda}(\Omega, \mathbb{G})}\right) \sup _{B_{4 r}\left(z_{0}\right)}|u| \cdot d\left(z, z_{0}\right)^{\alpha},
$$

where $\alpha=\min \{\beta,(1-\beta)(\lambda-Q+2)\}$. 


\section{Preliminary results}

In this section we recall some result about the fundamental solution and to the Green function $G_{0}$ for operators satisfying assumptions [H.1]-[H.2]; we then prove a lower bound for $G_{0}$. We end the section with some remarks on the Stummel-Kato class $S K\left(\Omega, \mathcal{L}_{0}\right)$.

In [11], Kogoj and Lanconelli prove the existence of a fundamental solution $\Gamma_{0}(z, \zeta)$ for the operators $\mathcal{L}_{0}$ satisfying conditions [H.1]-[H.2]. The main properties of $\Gamma_{0}$ are analogous to the properties of the heat kernel: $\Gamma_{0}$ is smooth in $\left\{(z, \zeta) \in \mathbb{R}^{N+1} \times \mathbb{R}^{N+1}: z \neq \zeta\right\} ; \Gamma_{0}(x, t, \xi, \tau) \geq 0$; for any $(x, t),(\xi, \tau) \in \mathbb{R}^{N+1}, \Gamma_{0}(x, t, \xi, \tau)>0$ if, and only if, $t>\tau$.

$\Gamma_{0}$ is invariant with respect to the translations of $\mathbb{G}$ :

$$
\Gamma_{0}(z, \zeta)=\Gamma_{0}\left(\zeta^{-1} \circ z, 0\right) \equiv \Gamma_{0}\left(\zeta^{-1} \circ z\right), \quad \text { for every } \quad z, \zeta \in \mathbb{R}^{N+1},
$$

and it is $\delta_{\lambda}$-homogeneous of degree $2-Q$ with respect to the dilations of $\mathbb{G}$ :

$$
\Gamma_{0}\left(\delta_{\lambda}(z)\right)=\lambda^{2-Q} \Gamma_{0}(z) \quad \text { for every } \quad z \in \mathbb{R}^{N+1}, \lambda>0 ;
$$

as a consequence we have that

$$
\lim _{|z| \rightarrow \infty} \Gamma_{0}(z)=0 ; \quad \limsup _{z \rightarrow 0} \Gamma_{0}(z)=+\infty \quad \text { and } \quad \Gamma_{0} \in L_{\mathrm{loc}}^{1}\left(\mathbb{R}^{N+1}\right)
$$

For every $\varphi \in C_{0}^{\infty}\left(\mathbb{R}^{N+1}\right)$ and $z \in \mathbb{R}^{N+1}$ we have

$$
\mathcal{L}_{0} \int_{\mathbb{R}^{N+1}} \Gamma_{0}(z, \zeta) \varphi(\zeta) d \zeta=-\varphi(z), \quad \int_{\mathbb{R}^{N+1}} \Gamma_{0}(z, \zeta) \mathcal{L}_{0} \varphi(\zeta) d \zeta=-\varphi(z)
$$

and $\mathcal{L}_{0} \Gamma_{0}(z, \cdot)=-\delta_{z}($ the Dirac measure centered at $z)$. Moreover

$$
\int_{\mathbb{R}^{N}} \Gamma_{0}(x, t) d x=1, \quad \text { for every } \quad t>0 .
$$

The function $\Gamma_{0}^{*}(z, \zeta) \equiv \Gamma_{0}(\zeta, z)$ is the fundamental solution of the adjoint operator $\mathcal{L}_{0}{ }^{*}$.

Since $\Gamma_{0}$ is a $\delta_{\lambda}$-homogeneous functions of degree $-Q+2$ and the derivatives $X_{j} \Gamma_{0}$, for $j=1, \ldots, m$, are $\delta_{\lambda}$-homogeneous functions of degree $-Q+1$, from the general theory of function spaces on homogeneous Lie groups (see for instance Folland [8, Proposition (1.15)]; see also Rothschild and Stein [24] for a more developed analysis of differential operators on Lie groups) it follows that there exist a positive constant $\widetilde{C}$ such that, for every 
$z_{1}, z_{2}, \zeta \in \mathbb{R}^{N+1}$, with $d\left(z_{1}, \zeta\right) \geq 2 d\left(z_{1}, z_{2}\right)$ we have

$$
\begin{aligned}
&\left|\Gamma_{0}\left(z_{1}, \zeta\right)-\Gamma_{0}\left(z_{2}, \zeta\right)\right| \leq \widetilde{C} \frac{d\left(z_{1}, z_{2}\right)}{d\left(z_{1}, \zeta\right)^{Q-1}}, \\
&\left|X_{j} \Gamma_{0}\left(z_{1}, \zeta\right)-X_{j} \Gamma_{0}\left(z_{2}, \zeta\right)\right| \leq \widetilde{C} \frac{d\left(z_{1}, z_{2}\right)}{d\left(z_{1}, \zeta\right)^{Q}}, \\
&\left|X_{j}^{(\zeta)} \Gamma_{0}\left(z_{1}, \zeta\right)-X_{j}^{(\zeta)} \Gamma_{0}\left(z_{2}, \zeta\right)\right| \leq \widetilde{C} \frac{d\left(z_{1}, z_{2}\right)}{d\left(z_{1}, \zeta\right)^{Q}},
\end{aligned}
$$

for $j=1, \ldots, m$ (the notation $X_{j}^{(\zeta)}$ means that the vector field $X_{j}$ acts on the variable $\zeta)$. Moreover, if we set for $f \in L^{p}\left(\mathbb{R}^{N+1}\right)$

$$
T_{f}(z)=\int_{\mathbb{R}^{N+1}} \Gamma_{0}(z, \zeta) f(\zeta) d \zeta
$$

we have (see $[8$, Theorem (5.14)]):

i) if $1<p<\frac{Q}{2}$, then $T_{f} \in L^{q}\left(\mathbb{R}^{N+1}\right)$, for $\frac{1}{q}=\frac{1}{p}-\frac{2}{Q}$, and

$$
\left\|T_{f}\right\|_{q} \leq C_{p}\|f\|_{p}
$$

ii) if $p>\frac{Q}{2}$, then

(3.6) $\left|T_{f}\left(z_{1}\right)-T_{f}\left(z_{2}\right)\right| \leq C_{p} d\left(z_{1}, z_{2}\right)^{\alpha}\|f\|_{p}, \quad$ for every $z_{1}, z_{2} \in \mathbb{R}^{N+1}$

for some positive constant $C_{p}$ and $\alpha=\min \left\{1,2-\frac{Q}{p}\right\}$.

Finally, for $j=1, \ldots, m$ we have

$$
X_{j} T_{f}(z)=\int_{\mathbb{R}^{N+1}} X_{j} \Gamma_{0}(z, \zeta) f(\zeta) d \zeta
$$

and, analogously,

i) if $1<p<Q$, then $X_{j} T_{f} \in L^{q}\left(\mathbb{R}^{N+1}\right)$, with $\frac{1}{q}=\frac{1}{p}-\frac{1}{Q}$, and

$$
\left\|X_{j} T_{f}\right\|_{q} \leq C_{p}\|f\|_{p}
$$

ii) if $p>Q$, then

(3.9) $\left|X_{j} T_{f}\left(z_{1}\right)-X_{j} T_{f}\left(z_{2}\right)\right| \leq C_{p} d\left(z_{1}, z_{2}\right)^{\alpha}\|f\|_{p}, \quad$ for $\alpha=1-\frac{Q}{p}$. 
Note that, by (3.5), formula (3.2) extends to

$$
\mathcal{L}_{0} \int_{\mathbb{R}^{N+1}} \Gamma_{0}(z, \zeta) f(\zeta) \psi(\zeta) d \zeta=-f(z) \psi(z)
$$

for any $f \in L_{\text {loc }}^{p}\left(\mathbb{R}^{N+1}\right)$, with $1<p<\frac{Q}{2}$, and any cut-off function $\psi$. We also recall that $T_{f}$ is continuous from $L^{1}\left(\mathbb{R}^{N+1}\right)$ to $L_{\text {weak }}^{\frac{Q-2}{Q}}\left(\mathbb{R}^{N+1}\right)$; more specifically, there exists a positive constant $C$ such that $\operatorname{meas}\left\{z \in \mathbb{R}^{N+1}: \mid T_{f}(z)>\alpha\right\} \leq\left(\frac{C}{\alpha}\right)^{\frac{Q-2}{Q}}\|f\|_{L^{1}\left(\mathbb{R}^{N+1}\right)}, \quad$ for every $\alpha>0$ (see [8, Prop. 1.10]) hence we will also use formula (3.10) for $f \in L_{\text {loc }}^{1}\left(\mathbb{R}^{N+1}\right)$.

We next prove a lower bound for the Green function $G_{0}$ for $\mathcal{L}_{0}$ :

Proposition 3.1. For any positive $R$ and $T$ and every $(\xi, \tau) \in \mathbb{R}^{N+1}$ and $\alpha \in] 0,1\left[\right.$ there exist $\left.\delta_{0}, \varepsilon \in\right] 0,1[$ such that

$$
G_{0}(x, t, y, \tau) \geq \frac{2 \varepsilon}{\operatorname{meas}\left(S_{R}(\xi, \tau)\right)}
$$

for every $\left.\delta \in] 0, \delta_{0}\right], y \in S_{\delta R}(\xi, \tau)$ and $(x, t) \in Q_{\delta R}(\xi, \tau, T)$, such that $t \geq$ $\tau+\alpha T$.

Proof. Thanks to the invariance of the operator with respect to the translations and the dilations of the Lie group $\mathbb{G}$, it is not restrictive to assume $(\xi, \tau)=(0,0)$ and $R=1$; we also denote $S=S_{1}(0,0)$. Aiming to prove that $G_{0}(0, t, 0,0)>0$, for every $\left.\left.t \in\right] 0, T\right]$, we recall $(2.13)$. We first note that $h$ is a bounded function in the set $\{(x, t, 0,0) \in \overline{Q(T)} \times\{(0,0)\}\}$. On the other hand $\Gamma_{0}(0, t)=t^{-\frac{Q-2}{2}} \Gamma_{0}(0,1)$ by $(3.1)$, then

$$
G_{0}(0, t, 0,0)=\Gamma_{0}(0, t)-h(0, t, 0,0) \rightarrow+\infty
$$

as $t \rightarrow 0+$. Then $G_{0}(0, t, 0,0)>0$ for any positive small $t$. Since $G_{0} \geq 0$ by the Bony's maximum principle ([3, Theorem 3.2]) $G_{0}(0, t, 0,0)>0$ for $t \in] 0, T]$. In order to prove our claim we let

$$
\varepsilon=\frac{1}{4} \operatorname{meas}(S) \min _{[\alpha T, T]} G_{0}(0, t, 0,0) ;
$$

it is not restrictive to suppose $\varepsilon<1$. Since $G_{0}$ is a continuous function, there exists $\left.\delta_{0} \in\right] 0,1[$ such that

$$
G_{0}(x, t, y, 0) \geq \frac{2 \varepsilon}{\operatorname{meas}(S)}
$$


for every $(x, t) \in Q_{\delta}(0,0, T)$, such that $t \geq \alpha T$ and $y \in S_{\delta}(0,0)$, with $\left.\delta \in] 0, \delta_{0}\right]$. This proves the claim for $(\xi, \tau)=(0,0)$ and $R=1$. The result in the general case follows by using the invariance with respect to the Lie group structure.

Remark 3.2. In the above proof we used the assumption (1.4) in order to apply the Bony's strong maximum principle along the segment $\{(0, t): 0 \leq$ $t \leq T\}$, which is a trajectory of the vector field $Y$.

If we remove the requirement (1.4), then the hypothesis [H.2] and (2.7) yield $Y(0)=b_{0} \cdot \nabla^{(2)}-\partial_{t}$, for some constant vector $b_{0}$ belonging to the second layer of the Lie algebra of $\mathbb{G}$. In this case, it is possible to adapt the proof of Proposition 3.1 by using the change of variable $(x, t) \mapsto\left(x-t b_{0}, t\right)$, however the assertion has to be stated according to the appropriate geometry.

We end this section with some further remarks about our definition of the Stummel-Kato class. We first recall the upper gaussian estimate for the fundamental solution provided by Kogoj and Lanconelli (see (5.1) in [11]), that allows us to establish whether a given function $\mathcal{V}$ does satisfy condition (1.11): for every $t>0, x \in \mathbb{R}^{N}$

$$
\Gamma_{0}(x, t) \leq \frac{C}{t^{\frac{Q-2}{2}}} \exp \left(-\frac{|x|_{\mathbb{G}}}{C t}\right)
$$

for some positive constant $C$.

We next observe that, unlike in the usual definition of the Stummel-Kato class, in formula (1.12) we integrate $\mathcal{V}$ on an unbounded set. A definition more similar to that one of the elliptic case should be given in terms of the following functions

$$
\begin{aligned}
& \widetilde{\eta}_{\mathcal{V}}(h)=\sup _{\substack{(x, t) \in \Omega \\
\Omega \cap Q_{h}\left(x, t, h^{2}\right)}} \Gamma_{0}(x, t, y, s)|\mathcal{V}(y, s)| d y d s, \\
& \widetilde{\eta}_{\mathcal{V}}^{*}(h)=\sup _{\substack{(y, s) \in \Omega \\
\Omega \cap Q_{h}^{*}\left(y, s, h^{2}\right)}} \int_{0}(x, t, y, s)|\mathcal{V}(x, t)| d x d t ;
\end{aligned}
$$

however, it turns out that $\widetilde{\eta}_{\mathcal{V}}$ and $\widetilde{\eta}_{\mathcal{V}}^{*}$ define the same class as $\eta_{\mathcal{V}}$ and $\eta_{\mathcal{V}}^{*}$.

Remark 3.3. We have that

$$
\begin{aligned}
& \lim _{h \rightarrow 0} \eta_{\mathcal{V}}(h)=0 \quad \Longleftrightarrow \quad \lim _{h \rightarrow 0} \widetilde{\eta}_{\mathcal{V}}(h)=0 ; \\
& \lim _{h \rightarrow 0} \eta_{\mathcal{V}}^{*}(h)=0 \quad \Longleftrightarrow \quad \lim _{h \rightarrow 0} \widetilde{\eta}_{\mathcal{V}}^{*}(h)=0 .
\end{aligned}
$$

One of the two implications is an easy consequence of the inequalities $\widetilde{\eta}_{\mathcal{V}}(h) \leq$ $\eta_{\mathcal{V}}(h)$ and $\widetilde{\eta}_{\mathcal{V}}^{*}(h) \leq \eta_{\mathcal{V}}^{*}(h)$. The other one easily follows from the homogeneity of $\Gamma_{0}$, with respect to the dilation of the Lie group, and from the absolute continuity of the integral. 
We next compare the spaces $S K\left(\Omega, \mathcal{L}_{0}\right)$ and the following Morrey spaces $L^{p, \lambda}(\Omega, \mathbb{G})$

Definition 3.4. Let $\Omega$ be an open subset of $\mathbb{R}^{N+1}$ and let $p, \lambda \in \mathbb{R}$ be such that $1 \leq p<\infty$ and $0 \leq \lambda \leq Q$. We say that a function $f \in L_{\text {loc }}^{p}(\Omega)$ belongs to the Morrey space $L^{p, \lambda}(\Omega, \mathbb{G})$ if $\|f\|_{L^{p, \lambda}(\Omega, \mathbb{G})}<\infty$, where

$$
\|f\|_{L^{p, \lambda}(\Omega, \mathbb{G})}=\left(\sup _{r>0, z \in \Omega} \frac{1}{r^{\lambda}} \int_{\Omega \cap B_{r}(z)}|f(w)|^{p} d w\right)^{\frac{1}{p}} .
$$

Although the class $S K\left(\Omega, \mathcal{L}_{0}\right)$ and the spaces $L^{p, \lambda}(\Omega, \mathbb{G})$ are defined analogously to the classic ones, we observe some substantial differences between them. In the case of elliptic equations we have

$$
L^{1, \lambda}(\Omega) \subseteq S K(\Omega) \subseteq L^{1, \mu}(\Omega), \quad 0<\mu \leq n-2<\lambda<n .
$$

An analogous result is true for the sum of the squares of the Hörmander fields, however in the case of parabolic (and degenerate parabolic) operators, we can prove the first inclusion, but the second one seems false (see Example 2.10 in [23]).

Proposition 3.5. We have

$$
\left.L^{1, \lambda}(\Omega, \mathbb{G}) \subseteq S K\left(\Omega, \mathcal{L}_{0}\right), \quad \text { for every } \lambda \in\right] Q-2, Q[.
$$

Proof. By using the homogeneity of the fundamental solution $\Gamma_{0}$ we find

$$
\int_{\Omega \cap Q_{h}\left(x, t, h^{2}\right)} \Gamma_{0}(x, t, w)|\mathcal{V}(w)| d w \leq c_{\lambda} h^{\lambda-Q+2} \frac{1}{h^{\lambda}} \int_{\Omega \cap B_{h}(x, t)}|\mathcal{V}(w)| d w,
$$

for every $\mathcal{V} \in L^{1, \lambda}(\Omega, \mathbb{G})$, and by Remark 3.3 this inequality yields the desired inclusion.

Since we are concerned with weak solutions to $\mathcal{L}_{\mathcal{V}} u=0$, we need a sufficient condition for the requirement $\mathcal{V} u \in L_{\text {loc }}^{1}$. We recall that, in the case of uniformly elliptic operators, $\mathcal{V} u \in L_{\text {loc }}^{1}(\Omega)$ provided that $u$ belongs to the space $H_{\text {loc }}^{1}(\Omega)$ (see Schechter [26]) and a similar condition holds for the sum of squares of Hörmander vector fields (see [5]). Here we prove that $\mathcal{V} u$ is locally integrable when $u$ belongs to the Sobolev-Folland-Stein space $W^{2,1}\left(\Omega, \mathcal{L}_{0}\right)$, namely if the following norm

$$
\|u\|_{W^{2,1}\left(\Omega, \mathcal{L}_{0}\right)}=\|u\|_{L^{1}(\Omega)}+\sum_{j=1}^{m}\left\|X_{j} u\right\|_{L^{1}(\Omega)}+\sum_{i, j=1}^{m}\left\|X_{i} X_{j} u\right\|_{L^{1}(\Omega)}+\|Y u\|_{L^{1}(\Omega)}
$$

is finite. 
Lemma 3.6. If $u \in W_{\text {loc }}^{2,1}\left(\Omega, \mathcal{L}_{0}\right)$ and $H, K$ are two compact sets such that $K \subset \subset H \subset \Omega$, then there exists a positive constant $C$, dependent only on $H, K$ and $\mathcal{V} \in S K\left(\Omega, \mathcal{L}_{0}\right)$, such that

$$
\int_{K}|\mathcal{V}(z) u(z)| d z \leq C\|u\|_{W^{2,1}\left(H, \mathcal{L}_{0}\right)} .
$$

Proof. We first claim that, for every $v \in C_{0}^{\infty}(\Omega)$, we have

$$
\int_{\Omega}|\mathcal{V}(z) v(z)| d z \leq C_{0}\|v\|_{W^{2,1}\left(\Omega, \mathcal{L}_{0}\right)}
$$

where $C_{0}$ is a positive constant dependent only on $\mathcal{V}$ and on the support of $v$. Indeed, if we denote by $H$ the support of $v$ then

$$
\begin{aligned}
& \int_{\Omega}|\mathcal{V}(z) v(z)| d z \leq \int_{H}|\mathcal{V}(z)|\left(\int_{H} \Gamma_{0}(z, \zeta)\left|\mathcal{L}_{0} v(\zeta)\right| d \zeta\right) d z \\
& \quad \leq \int_{H}\left|\mathcal{L}_{0} v(\zeta)\right| d \zeta \sup _{\eta \in H}\left(\int_{H}|\mathcal{V}(z)| \Gamma_{0}(z, \eta) d z\right) \leq \eta_{\mathcal{V}}^{*}\left(c_{H}\right)\|v\|_{W^{2,1}\left(\Omega, \mathcal{L}_{0}\right)}
\end{aligned}
$$

where $c_{H}=\max \{|t-\tau|:(x, t),(\xi, \tau) \in H\}$. This proves (3.16). The thesis follows from a standard density argument.

\section{The Green function for $\mathcal{L}_{\mathcal{V}}$}

In this section we use the parametrix method to prove the existence of a Green function $G$ for the operator $\mathcal{L}_{\mathcal{V}}$, related to any given cylinder $Q_{R}(\xi, \tau, T)$. We construct $G$ as a perturbation of $G_{0}$ :

$$
G(z, w)=G_{0}(z, w)+\int_{Q_{R}} G_{0}(z, \eta) \Phi(\eta, w) d \eta,
$$

for some unknown function $\Phi$. A formal argument, based on the fact that $\mathcal{L}_{0} G_{0}(z, w)=-\delta_{w}(z)$ and on the requirement that $\mathcal{L}_{\mathcal{V}} G(z, w)=-\delta_{w}(z)$ leads to the following Volterra equation for $\Phi$

$$
\Phi(z, \zeta)=\mathcal{V}(z) G_{0}(z, \zeta)+\int_{Q_{R}} \mathcal{V}(z) G_{0}(z, \eta) \Phi(\eta, \zeta) d \eta
$$

The successive approximation method then gives:

$$
G(z, w)=G_{0}(z, w)+\sum_{k=1}^{\infty} J_{k}(z, w)
$$


where

$$
\begin{aligned}
J_{1}(z, w) & =\int_{Q_{R}(\xi, \tau, T)} G_{0}(z, \eta) \mathcal{V}(\eta) G_{0}(\eta, w) d \eta \\
J_{k+1}(z, w) & =\int_{Q_{R}(\xi, \tau, T)} G_{0}(z, \eta) \mathcal{V}(\eta) J_{k}(\eta, w) d \eta .
\end{aligned}
$$

We will prove that these integrals $J_{k}$ are well defined, then the $L^{p}$ convergence of the series and we finally show that $G$ is a Green function for $\mathcal{L}_{\mathcal{V}}$. Aiming to unify the notations, in the sequel we will denote $J_{0}=G_{0}$ so that

$$
J_{1}(z, w)=\int_{Q_{R}(\xi, \tau, T)} G_{0}(z, \eta) \mathcal{V}(\eta) J_{0}(\eta, w) d \eta
$$

Lemma 4.1. The functions in (4.2) belong to $L^{p}\left(Q_{R}(\xi, \tau, T)\right)$ for every $p \in$ $\left[1, \frac{Q}{Q-2}\right)$ and there exists a positive constant $c_{p}$ such that

$$
\begin{gathered}
\left\|J_{k}(z, \cdot), L^{p}\left(Q_{R}(\xi, \tau, T)\right)\right\| \leq c_{p} \eta_{\mathcal{V}}^{*}(T)^{k} \\
\left\|J_{k}(\cdot, w), L^{p}\left(Q_{R}(\xi, \tau, T)\right)\right\| \leq c_{p} \eta_{\mathcal{V}}(T)^{k}
\end{gathered}
$$

for every $w, z \in Q_{R}(\xi, \tau, T)$. Moreover, $J_{k}(x, t, y, s)=0$ for every $t \leq s$. We can also write $J_{k+1}$ as

$$
J_{k+1}(z, w)=\int_{Q_{R}(\xi, \tau, T)} J_{k}(z, \eta) \mathcal{V}(\eta) G_{0}(\eta, w) d \eta
$$

Proof. We let $\widetilde{\mathcal{V}}(\eta)=|\mathcal{V}(\eta)|$ and define $\widetilde{J}_{k}$, by using formulas (4.2) with $\tilde{\mathcal{V}}$. Note that $\eta_{\mathcal{V}}(T)=\eta_{\tilde{\mathcal{V}}}(T)$ and $\eta_{\mathcal{V}}^{*}(T)=\eta_{\tilde{\mathcal{V}}}^{*}(T)$, then $\widetilde{\mathcal{V}} \in S K\left(Q_{T}, \mathcal{L}_{0}\right)$ if and only if $\mathcal{V} \in S K\left(Q_{T}, \mathcal{L}_{0}\right)$. We first prove the inequalities in (4.3) for the non-negative functions $\widetilde{J}_{k}$, the required estimates will follow from the trivial inequality $\left|J_{k}\right| \leq \widetilde{J}_{k}$.

Due to the fact that every $\widetilde{J}_{k}$ is non-negative, (4.4) is immediate. In order to prove the $L^{p}$ estimates for $\widetilde{J}_{k}$ we note that

$$
\begin{gathered}
\int_{Q_{R}(\xi, \tau, T)} G_{0}(z, \eta)|\mathcal{V}(\eta)| d \eta \leq \int_{Q_{R}(\xi, \tau, T)} \Gamma_{0}(z, \eta)|\mathcal{V}(\eta)| d \eta=\eta_{\mathcal{V}}(T), \\
\int_{Q_{R}(\xi, \tau, T)} G_{0}(\eta, w)|\mathcal{V}(\eta)| d \eta \leq \int_{Q_{R}(\xi, \tau, T)} \Gamma_{0}(\eta, w)|\mathcal{V}(\eta)| d \eta=\eta_{\mathcal{V}}^{*}(T),
\end{gathered}
$$

since $G_{0} \leq \Gamma_{0}$. 
We next define the sequences:

$$
\begin{aligned}
& s_{k}=\sup _{z \in Q_{R}(\xi, \tau, T)} \int_{Q_{R}(\xi, \tau, T)} \widetilde{J}_{k}(z, \eta)|\mathcal{V}(\eta)| d \eta, \\
& s_{k}^{*}=\sup _{\eta \in Q_{R}(\xi, \tau, T)} \int_{Q_{R}(\xi, \tau, T)}|\mathcal{V}(z)| \widetilde{J}_{k}(z, \eta) d z,
\end{aligned}
$$

and we prove the following inequalities

$$
s_{k} \leq \eta_{\mathcal{V}}(T)^{k+1}, \quad s_{k}^{*} \leq \eta_{\mathcal{V}}^{*}(T)^{k+1}
$$

by induction on $k$. For $k=1$ we have

$$
\begin{aligned}
& s_{1} \leq \sup _{z \in Q_{R}(\xi, \tau, T)} \int_{Q_{R}(\xi, \tau, T)} G_{0}(z, \zeta)|\mathcal{V}(\zeta)| \\
& \cdot\left(\sup _{w \in Q_{R}(\xi, \tau, T)} \int_{Q_{R}(\xi, \tau, T)} G_{0}(w, \eta)|\mathcal{V}(\eta)| d \eta\right) d \zeta \leq \eta_{\mathcal{V}}^{2}(T),
\end{aligned}
$$

by (4.5). The same argument and (4.2) give

$$
s_{k+1} \leq s_{k} \eta_{\mathcal{V}}(T)
$$

for any $k>1$, then the first inequality in (4.6) is proved. The proof of the second one is analogous.

To obtain the $L^{p}$ estimate for $\widetilde{J}_{k}$ we set, for $p \in\left[1, \frac{Q}{Q-2}\right)$ :

$$
\mathcal{T}=\left\{\varphi \in C_{0}^{\infty}\left(Q_{R}(\xi, \tau, T)\right): \varphi \geq 0, \quad\|\varphi\|_{L^{p^{\prime}}\left(Q_{R}(\xi, \tau, T)\right)} \leq 1\right\} .
$$

For any $\varphi \in \mathcal{T}$, we have

$$
\begin{aligned}
\int_{Q_{R}(\xi, \tau, T)} \widetilde{J}_{k+1}(z, w) \varphi(w) d w & =\int_{Q_{R}(\xi, \tau, T)} \widetilde{J}_{k}(z, \eta)|\mathcal{V}(\eta)|\left(\int_{Q_{R}(\xi, \tau, T)} G_{0}(\eta, w) \varphi(w) d w\right) d \eta \\
& \leq c_{p}\|\varphi\|_{L^{p^{\prime}}\left(Q_{R}(\xi, \tau, T)\right)} s_{k} \leq c_{p} \eta_{\mathcal{V}}^{*}(T)^{k+1},
\end{aligned}
$$

by (4.4) and (4.6), where

$$
c_{p}=\sup _{\eta \in Q_{R}(\xi, \tau, T)}\left\|\Gamma_{0}(\eta, \cdot)\right\|_{L^{p}\left(Q_{R}(\xi, \tau, T)\right)} .
$$

Thus

$$
\left\|\widetilde{J}_{k}(z, \cdot)\right\|_{L^{p}\left(Q_{R}(\xi, \tau, T)\right)}=\sup _{\varphi \in \mathcal{T}} \int_{Q_{R}(\xi, \tau, T)} \widetilde{J}_{k}(z, w) \varphi(w) d w \leq c_{p} \eta_{\mathcal{V}}^{*}(T)^{k}
$$

and the first inequality in (4.3) holds for every $k \in \mathbb{N}$. In the same way we obtain the second one. Since $\left|J_{k}(z, w)\right| \leq \widetilde{J}_{k}(z, w)$, the estimates (4.3) and the identity (4.4) also hold for every $J_{k}$, and the lemma is completely proved. 
Proposition 4.2. Let $T>0$ be such that $\eta_{\mathcal{V}}(T)<1$ and $\eta_{\mathcal{V}}^{*}(T)<1$. Then

i) for every $p \in\left[1, \frac{Q}{Q-2}\right)$ the series introduced in (4.1) converges in $L^{p}\left(Q_{R}(\xi, \tau, T)\right)$ and there exists a positive constant $c_{p}$ such that

$$
\|G(z, \cdot)\|_{L^{p}\left(Q_{R}(\xi, \tau, T)\right)} \leq c_{p} \sum_{k=0}^{\infty} \eta_{\mathcal{V}}(T)^{k} ;\|G(\cdot, w)\|_{L^{p}\left(Q_{R}(\xi, \tau, T)\right)} \leq c_{p} \sum_{k=0}^{\infty} \eta_{\mathcal{V}}^{*}(T)^{k}
$$

ii) $G(x, t, y, s)=0$ for $t \leq s$;

iii) the derivatives

$$
\begin{aligned}
& X_{j} G(\cdot, w)=X_{j} G_{0}(\cdot, w)+\sum_{k=1}^{\infty} \int_{Q_{R}(\xi, \tau, T)} X_{j} G_{0}(\cdot, \eta) \mathcal{V}(\eta) J_{k}(\eta, w) d \eta \\
& X_{j} G(z, \cdot)=X_{j} G_{0}(z, \cdot)+\sum_{k=1}^{\infty} \int_{Q_{R}(\xi, \tau, T)} J_{k}(z, \eta) \mathcal{V}(\eta) X_{j} G_{0}(\eta, \cdot) d \eta
\end{aligned}
$$

are defined as elements of the space $L_{\mathrm{loc}}^{p}\left(Q_{R}(\xi, \tau, T)\right)$ for any $p \in$ $\left[1, \frac{Q}{Q-1}\right)$ and, for every compact set $K \subset Q_{R}(\xi, \tau, T)$, there exists a positive constant $\bar{c}_{p}$ such that

$$
\left\|X_{j} G(\cdot, w)\right\|_{L^{p}(K)} \leq \bar{c}_{p} \sum_{k=0}^{\infty} \eta_{\mathcal{V}}(T)^{k}, \quad\left\|X_{j} G(z, \cdot)\right\|_{L^{p}(K)} \leq \bar{c}_{p} \sum_{k=0}^{\infty} \eta_{\mathcal{V}}^{*}(T)^{k},
$$

for $j=1, \ldots, m$;

iv) for every $(x, t) \in Q_{R}(\xi, \tau, T)$,

$$
\int_{S_{R}(\xi, \tau)}|G(x, t, y, \tau)| d y \leq \sum_{k=1}^{\infty} \eta_{\mathcal{V}}(T)^{k} ; \int_{S_{R}(\xi, \tau, T)}|G(y, \tau+T, x, t)| d y \leq \sum_{k=1}^{\infty} \eta_{\mathcal{V}}^{*}(T)^{k} ;
$$

v) for every $z \in Q_{R}(\xi, \tau, T)$, we have

$$
\int_{Q_{R}(\xi, \tau, T)}|G(z, w) \mathcal{V}(w)| d w \leq \sum_{k=1}^{\infty} \eta_{\mathcal{V}}(T)^{k}, \quad \int_{Q_{R}(\xi, \tau, T)}|\mathcal{V}(\zeta) G(\zeta, z)| d \zeta \leq \sum_{k=1}^{\infty} \eta_{\mathcal{V}}^{*}(T)^{k} .
$$

Proof. Assertions $i$ ) and $i$ ) are direct consequences of Lemma 4.1. In order to prove $i i i$ ), we show that the series

$$
\sum_{k=1}^{\infty} \int_{Q_{R}(\xi, \tau, T)} X_{j} G_{0}(\cdot, \eta) \mathcal{V}(\eta) J_{k}(\eta, w) d \eta
$$

is convergent in $L_{\mathrm{loc}}^{p}\left(Q_{R}(\xi, \tau, T)\right)$. 
Let $K$ be a compact subset of $Q_{R}(\xi, \tau, T)$; for any $\varphi \in \mathcal{T}$, such that $\operatorname{supp}(\varphi) \subset K$, we have

$$
\begin{aligned}
\int_{K}\left(\int_{Q_{R}(\xi, \tau, T)}\right. & \left.X_{j} G_{0}(z, \eta) \mathcal{V}(\eta) J_{k}(\eta, w) d \eta\right) \varphi(z) d z \\
& \leq \bar{c}_{p}\|\varphi\|_{L^{p^{\prime}(K)}} \sup _{w \in Q_{R}(\xi, \tau, T)} \int_{Q_{R}(\xi, \tau, T)}|\mathcal{V}(\eta)| \widetilde{J}_{k}(\eta, w) d \eta \\
& \leq \bar{c}_{p} \eta_{\mathcal{V}}^{*}(T)^{k+1}
\end{aligned}
$$

by (4.6), where

$$
\bar{c}_{p}=\sup _{\eta \in Q_{R}(\xi, \tau, T)}\left\|X_{j} G_{0}(\cdot, \eta)\right\|_{L^{p}(K)} .
$$

Hence

$$
\begin{aligned}
& \left\|\int_{Q_{R}(\xi, \tau, T)} X_{j} G_{0}(\cdot, \eta) \mathcal{V}(\eta) J_{k}(\eta, w) d \eta\right\|_{L^{p}(K)} \\
& \quad=\sup _{\varphi \in \mathcal{T}} \int_{K}\left(\int_{Q_{R}(\xi, \tau, T)} X_{j} G_{0}(z, \eta) \mathcal{V}(\eta) J_{k}(\eta, w) d \eta\right) \varphi(z) d z \\
& \leq \bar{c}_{p} \eta_{\mathcal{V}}^{*}(T)^{k+1} .
\end{aligned}
$$

This proves the first identity in (4.7) and the estimate

$$
\left\|X_{j} G(z, \cdot)\right\|_{L^{p}(K)} \leq \bar{c}_{p} \sum_{k=0}^{\infty} \eta_{\mathcal{V}}^{*}(T)^{k} .
$$

The same argument gives the second identity and the corresponding estimate.

In order to prove $i v$ ), we note that, for every $k \in \mathbb{N}$,

$$
\begin{aligned}
\mid \int_{S_{R}(\xi, \tau)} & J_{k}(x, t, y, \tau) d y \mid \\
& \leq\left|\int_{Q_{R}(\xi, \tau, T)} J_{k-1}(x, t, \eta) \mathcal{V}(\eta)\left(\int_{S_{R}(\xi, \tau)} G_{0}(\eta, y, \tau) d y\right) d \eta\right| \\
& \leq \eta_{\mathcal{V}}^{k}(T),
\end{aligned}
$$

by (4.6). This proves the first estimate, the proof of the second one is analogous.

Finally, $v$ ) is an immediate consequence of (4.6). This concludes the proof of Proposition 4.2. 
Corollary 4.3. The function $G$ defined in (4.1) is solution, in the distribution sense, of $\mathcal{L}_{\mathcal{V}} G(\cdot, \zeta)=-\delta_{\zeta}, \mathcal{L}_{\mathcal{V}}{ }^{*} G(z, \cdot)=-\delta_{z}$. Namely: $G(\cdot, \zeta)$, $X_{j} G(\cdot, \zeta) \in L^{p}\left(Q_{R}(\xi, \tau, T)\right)$, for some $p>1$ and for $j=1, \ldots, m, G \mathcal{V} \in$ $L^{1}\left(Q_{R}(\xi, \tau, T)\right)$ and $\forall \varphi \in C_{0}^{\infty}\left(Q_{R}(\xi, \tau, T)\right)$, we have

$$
\begin{gathered}
\int_{Q_{R}(\xi, \tau, T)}\left(\sum_{j=1}^{m} X_{j} G(z, \zeta) X_{j} \varphi(z)+G(z, \zeta) Y \varphi(z)-G(z, \zeta) \mathcal{V}(z) \varphi(z)\right) d z=\varphi(\zeta), \\
\int_{Q_{R}(\xi, \tau, T)}\left(\sum_{j=1}^{m} X_{j} G(z, \zeta) X_{j} \varphi(\zeta)-G(z, \zeta) Y \varphi(\zeta)-G(z, \zeta) \mathcal{V}(\zeta) \varphi(\zeta)\right) d \zeta=\varphi(z) .
\end{gathered}
$$

Proof. Since $G_{0}$ is the Green function of $\mathcal{L}_{0}$, we have

$$
\int_{Q_{R}(\xi, \tau, T)}\left(\sum_{j=1}^{m} X_{j} G_{0}(z, \zeta) X_{j} \varphi(z)+G_{0}(z, \zeta) Y \varphi(z)\right) d z=\varphi(\zeta),
$$

for every $\varphi \in C_{0}^{\infty}\left(Q_{R}(\xi, \tau, T)\right)$. For any $k \in \mathbb{N}$, we multiply the above identity by $\mathcal{V}(\zeta) J_{k-1}(\zeta, w)$ and integrate on $Q_{R}(\xi, \tau, T)$; we find

$$
\begin{aligned}
& \int_{Q_{R}(\xi, \tau, T)}\left(\sum_{j=1}^{m} X_{j} \varphi(z) \int_{Q_{R}(\xi, \tau, T)} X_{j} G_{0}(z, \zeta) \mathcal{V}(\zeta) J_{k-1}(\zeta, w) d \zeta+\right. \\
&\left.Y \varphi(z) \int_{Q_{R}(\xi, \tau, T)} G_{0}(z, \zeta) \mathcal{V}(\zeta) J_{k-1}(\zeta, w) d \zeta\right) d z=\int_{Q_{R}(\xi, \tau, T)} \varphi(\zeta) \mathcal{V}(\zeta) J_{k-1}(\zeta, w) d \zeta
\end{aligned}
$$

and the first identity follows from the definition (4.1), (4.2) and from (4.7). In analogue way we can proceed for the second equality.

Proposition 4.4. Let $T>0$ be such that $\eta_{\mathcal{V}}(T)<1$ and $\eta_{\mathcal{V}}^{*}(T)<1$. Then, for any $(\xi, \tau) \in \mathbb{R}^{N+1}$ the function $G$ defined by (4.1) is the Green function for the Cauchy-Dirichlet problem (2.16) related to $Q_{R}(\xi, \tau, T)$.

Moreover $G^{*}(w, z)=G(z, w)$ is the Green function for the CauchyDirichlet problem

$$
\begin{cases}\mathcal{L}_{\mathcal{V}}^{*} v=g & \text { in } Q_{R}(\xi, \tau, T) \\ v=0 & \text { in } \partial_{r}^{*} Q_{R}(\xi, \tau, T)\end{cases}
$$

with $g \in C_{0}\left(Q_{R}(\xi, \tau, T)\right)$, namely the function

$$
v(y, s)=-\int_{Q_{R}(\xi, \tau, T)} G^{*}(y, s, z) g(z) d z
$$

is a weak solution to $\mathcal{L}_{\mathcal{V}}{ }^{*} v=g$ in $Q_{R}(\xi, \tau, T)$ and attains the boundary data by continuity $\left(\right.$ in $(4.8) \partial_{r}^{*} Q_{R}(\xi, \tau, T)=(\xi, \tau) \circ \delta_{R}\left(\partial_{r}^{*} Q\left(R^{-2} T\right)\right)$, where $\left.\partial_{r}^{*} Q(T)=S(T) \cup(\partial O \times[0, T])\right)$. 
Proof. As said in Section 2, $G$ is a Green function for the Cauchy-Dirichlet problem (2.16) if, for any $f \in C_{0}\left(Q_{R}(\xi, \tau, T)\right)$, the function

$$
u(z)=-\int_{Q_{R}(\xi, \tau, T)} G(z, \zeta) f(\zeta) d \zeta
$$

is a weak solution to $\mathcal{L}_{\mathcal{V}} u=f$ in $Q_{R}(\xi, \tau, T)$ and attains the boundary data by continuity. The fact that $u$ solves $\mathcal{L}_{\mathcal{V}} u=f$ is a direct consequence of Corollary 4.3.

In order to verify that $u$ continuously vanishes at $\partial_{r} Q_{R}(\xi, \tau, T)$ we first note that

$$
\mathcal{L}_{0} u(z)=f(z)-\mathcal{V}(z) u(z)
$$

then

$$
u(z)=-\int_{Q_{R}(\xi, \tau, T)} G_{0}(z, \eta) f(\eta) d \eta+\int_{Q_{R}(\xi, \tau, T)} G_{0}(x, t, \eta) \mathcal{V}(\eta) u(\eta) d \eta
$$

for every $z \in Q_{R}(\xi, \tau, T)$. Since the function

$$
u_{0}(z)=-\int_{Q_{R}(\xi, \tau, T)} G_{0}(z, \eta) f(\eta) d \eta
$$

is a solution to the boundary value problem $(2.12)$ (which is related to $\mathcal{L}_{0}$ ) it is known that it continuously vanishes at $\partial_{r} Q_{R}(\xi, \tau, T)$. Hence, we have to show that

$$
\lim _{(x, t) \rightarrow\left(x_{0}, t_{0}\right)} \int_{Q_{R}(\xi, \tau, T)} G_{0}(x, t, \eta) \mathcal{V}(\eta) u(\eta) d \eta=0,
$$

for every $\left(x_{0}, t_{0}\right) \in \partial_{r} Q_{R}(\xi, \tau, T)$.

In order to prove (4.9) we observe that $u$ is a bounded function, by Proposition 4.2 (i). Let us first consider a point $\left(x_{0}, t_{0}\right) \in S_{R}(\xi, \tau)$. Since $\mathcal{V}$ belongs to the Stummel-Kato class, we have

$$
\left|\int_{Q_{R}(\xi, \tau, T)} G_{0}(x, t, \eta) \mathcal{V}(\eta) u(\eta) d \eta\right| \leq\|u\|_{\infty} \eta_{\mathcal{V}}(t) \rightarrow 0 \quad \text { as } t \rightarrow 0^{+} .
$$

This proves that $u(x, t) \rightarrow 0$ as $(x, t) \rightarrow\left(x_{0}, t_{0}\right)$, for any $\left(x_{0}, t_{0}\right) \in S_{R}(\xi, \tau)$.

We next consider a point $\left(x_{0}, t_{0}\right) \in M_{R}(\xi, \tau, T)$. For every positive $\varepsilon$ there exists a $\delta>0$ such that

$$
\left|\int_{(y, s) \in Q_{R}(\xi, \tau, T): t_{0}-\delta<s<t_{0}} G_{0}(x, t, y, s) \mathcal{V}(y, s) u(y, s) d y d s\right| \leq\|u\|_{\infty} \eta_{\mathcal{V}}(\delta)<\varepsilon
$$


$\forall(x, t) \in Q_{R}(\xi, \tau, T)$, since $\mathcal{V} \in S K\left(\Omega, \mathcal{L}_{0}\right)$. Moreover there exists a positive constant $\widetilde{H}$ such that $G_{0}(x, t, y, s) \leq \widetilde{H}$, for every $(x, t),(y, s) \in Q_{R}(\xi, \tau, T)$ such that $s<t_{0}-\delta$ and $t>t_{0}-\delta / 2$. Hence

$$
\lim _{(x, t) \rightarrow\left(x_{0}, t_{0}\right)} \int_{\eta \in Q_{R}(\xi, \tau, T): s<t_{0}-\delta} G_{0}(x, t, \eta) \mathcal{V}(\eta) u(\eta) d \eta=0,
$$

that proves (4.9). This completes the proof that $u(x, t) \rightarrow 0$ as $(x, t) \rightarrow$ $\left(x_{0}, t_{0}\right)$ for every $\left(x_{0}, t_{0}\right) \in \partial_{r} Q_{R}(\xi, \tau, T)$, thus $G$ is a Green function for $\mathcal{L}_{\mathcal{V}}$ in $Q_{R}(\xi, \tau, T)$.

The proof that $G^{*}$ is a Green function for $\mathcal{L}_{\mathcal{V}}{ }^{*}$ in $Q_{R}(\xi, \tau, T)$ is analogous and will be omitted.

We next prove a lower bound for $G$ analogous to Proposition 3.1.

Proposition 4.5. For every $\left.\alpha_{0} \in\right] 0,1\left[\right.$ there exist $\left.\varepsilon, \delta_{0} \in\right] 0,1\left[\right.$, and $R_{0}>0$ such that, if $\left.\left.\left.R \in] 0, R_{0}\right], \delta \in\right] 0, \delta_{0}\right]$, and $G$ is the Green function related to $Q_{R}\left(\xi, \tau, R^{2}\right)$ then

$$
G(x, t, y, \tau) \geq \frac{\varepsilon}{\operatorname{meas}\left(S_{R}(\xi, \tau)\right)}
$$

for every $y \in S_{\delta R}(\xi, \tau)$ and for every $(x, t) \in Q_{\delta R}\left(\xi, \tau, R^{2}\right)$, such that $t \geq$ $\tau+\alpha_{0} R^{2}$

Proof. We claim that there exists a positive constant $c$ such that

$$
\left|J_{k}(x, t, \bar{x}, \bar{t})\right| \leq \frac{c}{(t-\bar{t})^{\frac{Q-2}{2}}}\left(\eta_{\mathcal{V}}(t-\bar{t})+\eta_{\mathcal{V}}^{*}(t-\bar{t})\right)^{k}
$$

for every $k \in \mathbb{N}$ and any $(x, t),(\bar{x}, \bar{t}) \in Q_{R}\left(\xi, \tau, R^{2}\right)$. As a consequence, from (4.1) and Proposition 3.1 we get

$$
\begin{aligned}
G(x, t, y, \tau) & \geq G_{0}(x, t, y, \tau)-\frac{c}{(t-\tau)^{\frac{Q-2}{2}}} \sum_{k=1}^{\infty}\left(\eta_{\mathcal{V}}(t-\tau)+\eta_{\mathcal{V}}^{*}(t-\tau)\right)^{k} \\
& \geq \frac{2 \varepsilon}{\operatorname{meas}\left(S_{R}(\xi, \tau)\right)}-\frac{c}{(t-\tau)^{\frac{Q-2}{2}}} \sum_{k=1}^{\infty}\left(\eta_{\mathcal{V}}(t-\tau)+\eta_{\mathcal{V}}^{*}(t-\tau)\right)^{k},
\end{aligned}
$$

for any $y \in S_{\delta R}(\xi, \tau)$ and for every $(x, t) \in Q_{\delta R}\left(\xi, \tau, R^{2}\right)$, such that $t \geq$ $\tau+\alpha_{0} R^{2}$. Moreover, since meas $\left(S_{R}(\xi, \tau)\right)=R^{Q-2}$ meas $(S)$ and $\alpha_{0} R^{2} \leq$ $t-\tau \leq R^{2}$, the above inequality gives

$G(x, t, y, \tau) \geq \frac{2 \varepsilon}{\operatorname{meas}\left(S_{R}(\xi, \tau)\right)}-\frac{c^{\prime}}{\operatorname{meas}\left(S_{R}(\xi, \tau)\right)} \sum_{k=1}^{\infty}\left(\eta_{\mathcal{V}}(t-\tau)+\eta_{\mathcal{V}}^{*}(t-\tau)\right)^{k}$, for some positive constant $c^{\prime}$. The claim then follows by choosing $R_{0}$ suitably small. 
We next prove (4.10) by induction. We first recall (3.11), then

$$
G_{0}(x, t, y, s) \leq \Gamma_{0}(x, t, y, s) \leq \frac{C}{(t-s)^{\frac{Q-2}{2}}},
$$

for every $(x, t),(y, s) \in Q_{R}\left(\xi, \tau, R^{2}\right)$. Since

$$
\begin{aligned}
J_{1}(x, t, \bar{x}, \tau)= & \int_{\tau}^{\frac{t+\tau}{2}} \int_{S_{R}(\xi, \tau, s)} G_{0}(x, t, y, s) \mathcal{V}(y, s) G_{0}(y, s, \bar{x}, \tau) d y d s+ \\
& +\int_{\frac{t+\tau}{2}}^{t} \int_{S_{R}(\xi, \tau, s)} G_{0}(x, t, y, s) \mathcal{V}(y, s) G_{0}(y, s, \bar{x}, \tau) d y d s
\end{aligned}
$$

we have

$$
\begin{aligned}
\left|J_{1}(x, t, \bar{x}, \tau)\right| \leq & \frac{C}{\left(\frac{t-\tau}{2}\right)^{\frac{Q-2}{2}}} \int_{\tau}^{\frac{t+\tau}{2}} \int_{S_{R}(\xi, \tau, s)}|\mathcal{V}(y, s)| \Gamma_{0}(y, s, \bar{x}, \tau) d y d s \\
& +\frac{C}{\left(\frac{t-\tau}{2}\right)^{\frac{Q-2}{2}}} \int_{\frac{t+\tau}{2}}^{t} \int_{S_{R}(\xi, \tau, s)} \Gamma_{0}(x, t, y, s)|\mathcal{V}(y, s)| d y d s
\end{aligned}
$$

so that (4.10) follows for $k=1$.

For $k>1$ we argue analogously: we write

$$
\begin{aligned}
J_{k+1}(x, t, \bar{x}, \tau)= & \int_{\tau}^{\frac{t+\tau}{2}} \int_{S_{R}(\xi, \tau, s)} J_{k}(x, t, y, s) \mathcal{V}(y, s) G_{0}(y, s, \bar{x}, \tau) d y d s \\
& +\int_{\frac{t+\tau}{2}}^{t} \int_{S_{R}(\xi, \tau, s)} J_{k}(x, t, y, s) \mathcal{V}(y, s) G_{0}(y, s, \bar{x}, \tau) d y d s
\end{aligned}
$$

and we use (4.6) in the second integral. This completes the proof.

\section{Proof of the main results}

In this section we prove the main results of this paper. As said in the introduction, the main difficulty is in the fact that $\mathcal{V}$ is unbounded, then we cannot rely on the usual maximum principle. To overcome this problem, we first prove Proposition 2.3 and an uniqueness result for bounded solutions, then we prove the Harnack inequality (Theorem 2.2) for a bounded function $\mathcal{V}$, with the constant $M$ depending on $\eta_{\mathcal{V}}$ and $\eta_{\mathcal{V}}^{*}$, but not on the $L^{\infty}$ norm of $\mathcal{V}$. We finally remove the hypotheses of boundedness from $u$ and $\mathcal{V}$, by using a technique due to Zhang [29]. We consider the sequence of operators

$$
\mathcal{L}_{\mathcal{V}_{m}}=\mathcal{L}_{0}+\mathcal{V}_{m}
$$


where

$$
\mathcal{V}_{m}(x, t)= \begin{cases}-m & \text { if } \mathcal{V}(x, t) \leq-m \\ \mathcal{V}(x, t) & \text { if }-m<\mathcal{V}(x, t)<m \\ m & \text { if } \mathcal{V}(x, t) \geq m\end{cases}
$$

and we approximate the solution $u$ to $\mathcal{L}_{\mathcal{V}}=0$ by a sequence $u_{m}$ of solutions to $\mathcal{L}_{\mathcal{V}_{m}}=0$. Since $\eta_{\mathcal{V}_{m}}(T) \leq \eta_{\mathcal{V}}(T)$, and $\eta_{\mathcal{V}_{m}}^{*}(T) \leq \eta_{\mathcal{V}}^{*}(h)$, the Harnack inequality for bounded solutions extends to $u$.

Lemma 5.1. If $u$ is a bounded weak solution of $\mathcal{L}_{\mathcal{V}} u=0$ in $\Omega$, with $\mathcal{V} \in$ $S K\left(\Omega, \mathcal{L}_{0}\right)$, then $u$ is continuous. Moreover, for any $\left.\beta \in\right] 0,1[$ there exists a positive constant $C_{\beta}$, dependent only on $\mathcal{L}_{0}$ and $\beta$, such that

$$
\left|u(z)-u\left(z_{0}\right)\right| \leq\left(C_{\beta} d\left(z, z_{0}\right)^{\beta}+2 \eta_{\mathcal{V}}\left(5 c^{2} d\left(z, z_{0}\right)^{1-\beta}\right)\right) \sup _{B_{4 r}\left(z_{0}\right)}|u|
$$

for every $\left.z_{0} \in \Omega, r \in\right] 0,1\left[\right.$ such that $B_{4 r}\left(z_{0}\right) \subset \Omega$ and for every $z \in B_{r_{\beta}}\left(z_{0}\right)$ (where $r_{\beta}=r^{\frac{1}{1-\beta}}$ and $c$ is the constant in (2.3)).

If $\mathcal{V} \in L^{1, \lambda}(\Omega, \mathbb{G})$ with $\left.\lambda \in\right] Q-2, Q[$, then

$$
\left|u(z)-u\left(z_{0}\right)\right| \leq C_{\beta}\left(1+\|\mathcal{V}\|_{L^{1, \lambda}(\Omega, \mathbb{G})}\right) \sup _{B_{4 r}\left(z_{0}\right)}|u| \cdot d\left(z, z_{0}\right)^{\alpha},
$$

where $\alpha=\min \{\beta,(1-\beta)(\lambda-Q+2)\}$.

Proof. Let $z_{0} \in \Omega, r \in(0,1)$ be such that $B_{4 r}\left(z_{0}\right) \subset \Omega$ and let $z \in B_{r_{\beta}}\left(z_{0}\right)$. We choose $\varrho=2 d\left(z_{0}, z\right)^{1-\beta}$ and a function $\varphi \in C_{0}^{\infty}\left(B_{2 \varrho}\left(z_{0}\right)\right)$ such that $\varphi \equiv 1$ in $B_{\varrho}\left(z_{0}\right)$ and that $\left|X_{j} \varphi\right| \leq \frac{c}{\varrho},\left|X_{i} X_{j} \varphi\right| \leq \frac{c}{\varrho^{2}}$, for $i, j=1, \ldots, m$ and $|Y \varphi| \leq \frac{c}{\varrho^{2}}$, for some positive constant $c$ only depending on the operator $\mathcal{L}_{0}$.

Since $\varrho \leq 2 r$, we have $B_{2 \varrho}\left(z_{0}\right) \subset B_{4 r}\left(z_{0}\right) \subset \Omega$ and $(\varphi u): B_{2 \varrho}\left(z_{0}\right) \rightarrow \mathbb{R}$ satisfies

$$
\mathcal{L}_{0}(\varphi u)=\sum_{j=1}^{m} X_{j}^{2}(\varphi u)+Y(\varphi u)=\varphi \mathcal{L}_{0} u+u \mathcal{L}_{0} \varphi+2 \sum_{j=1}^{m} X_{j} \varphi X_{j} u .
$$

By the representation formula (3.10) we have that

$$
\begin{aligned}
u(z)= & -\int_{\mathbb{R}^{N+1}} \Gamma_{0}(z, \zeta) \mathcal{L}_{0} \varphi(\zeta) u(\zeta) d \zeta-2 \sum_{j=1}^{m} \int_{\mathbb{R}^{N+1}} \Gamma_{0}(z, \zeta)\left\langle X_{j} \varphi, X_{j} u\right\rangle d \zeta \\
& +\int_{\mathbb{R}^{N+1}} \Gamma_{0}(z, \zeta) \mathcal{V}(\zeta) u(\zeta) \varphi(\zeta) d \zeta \\
= & A_{1}(z)+A_{2}(z)+A_{3}(z), \quad \forall z \in B_{\varrho}\left(z_{0}\right) .
\end{aligned}
$$


We explicitly remark that $d\left(z_{0}, \zeta\right) \geq 2 d\left(z_{0}, z\right)$, for every $\zeta \in B_{2 \varrho}\left(z_{0}\right) \backslash B_{\varrho}\left(z_{0}\right)$. From the first inequality in (3.3) we estimate the two terms in $A_{1}$ as follows

$$
\begin{aligned}
& \left|A_{1}(z)-A_{1}\left(z_{0}\right)\right| \leq \int_{\mathbb{R}^{N+1}}\left|\Gamma_{0}(z, \zeta)-\Gamma_{0}\left(z_{0}, \zeta\right)\right| \cdot\left|\mathcal{L}_{0} \varphi(\zeta) u(\zeta)\right| d \zeta \\
& \quad \leq \widetilde{C} \sup _{B_{2 \varrho}\left(z_{0}\right)}\left|u \mathcal{L}_{0} \varphi\right| \int_{B_{2 \varrho}\left(z_{0}\right) \backslash B_{\varrho}\left(z_{0}\right)} \frac{d\left(z_{1}, z_{2}\right)}{d\left(z_{1}, \zeta\right)^{Q-1}} d \zeta \leq C_{\beta} d\left(z, z_{0}\right)^{\beta} \sup _{B_{2 \varrho}\left(z_{0}\right)}|u|,
\end{aligned}
$$

for some positive constant $C_{\beta}$ depending on $\widetilde{C}$ in (3.3), on $\mathcal{L}_{0} \varphi$ and on $\beta$.

We next consider $A_{2}$. We integrate by parts

$$
\begin{aligned}
& A_{2}(z)=2 \sum_{j=1}^{m} \int_{\mathbb{R}^{N+1}} X_{j}^{(\zeta)}\left(\Gamma_{0}(z, \zeta) X_{j} \varphi(\zeta)\right) u(\zeta) d \zeta \\
& =2 \sum_{j=1}^{m} \int_{\mathbb{R}^{N+1}} \Gamma_{0}(z, \zeta) X_{j}^{2} \varphi(\zeta) u(\zeta) d \zeta+2 \sum_{j=1}^{m} \int_{\mathbb{R}^{N+1}} X_{j}^{(\zeta)} \Gamma_{0}(z, \zeta) X_{j} \varphi(\zeta) u(\zeta) d \zeta,
\end{aligned}
$$

(as in (3.3), the notation $X_{j}^{(\zeta)}$ means that the vector field $X_{j}$ acts on the variable $\zeta$ ). We then estimate the first sum by the same argument as $A_{1}$; for the second one we use the third inequality in (3.3).

We finally consider $A_{3}$. Let us first observe that, in view of (2.3), we have $d(z, \zeta) \leq c^{2}\left(d\left(z, z_{0}\right)+2 \varrho\right) \leq 5 c^{2} d\left(z_{0}, z\right)^{1-\beta}$ for every $\zeta \in \operatorname{supp}(\varphi)$, and $z \in B_{r}\left(z_{0}\right)$. Moreover, if $\zeta=(\xi, \tau)$ and $z=(x, t)$, then $|t-\tau| \leq d^{2}(z, \zeta)$, so that

$$
\left|A_{3}(z)\right| \leq \sup _{B_{2 \varrho}\left(z_{0}\right)}|u| \int_{B_{2 \varrho}\left(z_{0}\right)} \Gamma_{0}(z, \zeta)|\mathcal{V}(\zeta)| d \zeta \leq \sup _{B_{2 \varrho}\left(z_{0}\right)}|u| \cdot \eta_{\mathcal{V}}\left(5 c^{2} d\left(z_{0}, z\right)^{1-\beta}\right),
$$

for every $z \in B_{\varrho}\left(z_{0}\right)$. This proves the first claim of Lemma 5.1. The second assertion directly follows from Proposition 3.5 (see (3.14)).

We next prove a uniqueness result for Cauchy-Dirichlet problem (2.16).

Lemma 5.2. If $u$ is a bounded solution to the problem

$$
\begin{cases}\mathcal{L}_{\mathcal{V}} u=0 & \text { in } Q_{R}(\xi, \tau, T) \\ u=0 & \text { in } \partial_{r} Q_{R}(\xi, \tau, T)\end{cases}
$$

then $u \equiv 0$.

Proof. By the maximum principle, if $u$ and $v$ are weak solutions of the problem

$$
\begin{cases}\mathcal{L}_{0} u=f & \text { in } Q_{R}(\xi, \tau, T) \\ u=0 & \text { in } \partial_{r} Q_{R}(\xi, \tau, T)\end{cases}
$$

with $f \in L^{1}\left(Q_{R}(\xi, \tau, T)\right)$, then $u \equiv v$. 
Hence, if $u$ is a solution of the non-homogeneous problem

$$
\begin{cases}\mathcal{L}_{0} u+\mathcal{V} u=f & \text { in } Q_{R}(\xi, \tau, T) \\ u=0 & \text { in } \partial_{r} Q_{R}(\xi, \tau, T)\end{cases}
$$

with $f \in L^{1}\left(Q_{R}(\xi, \tau, T)\right)$, then $u$ is almost everywhere equal to

$$
v(z)=\int_{Q_{R}(\xi, \tau, T)} G_{0}(z, \zeta)(\mathcal{V}(\zeta) u(\zeta)-f(\zeta)) d \zeta .
$$

Suppose now that $u$ is a solution of the homogeneous problem (5.3). We then have

$$
u(x, t)=\int_{Q_{R}(\xi, \tau, T)} G_{0}(x, t, y, s) \mathcal{V}(y, s) u(y, s) d y d s .
$$

for every $(x, t) \in Q_{R}(\xi, \tau, h)$. Then recalling that $G_{0}(x, t, y, s)=0$ for $t \leq s$ we have for $t<\tau+\delta$

$$
\|u\|_{L^{\infty}\left(Q_{R}(\xi, \tau, \delta)\right)} \leq \eta_{V}(\delta)\|u\|_{L^{\infty}\left(Q_{R}(\xi, \tau, \delta)\right)} .
$$

Thus, if we choose $\delta$ such that $\eta_{V}(\delta)<1$, we have $u \equiv 0$ in $Q_{R}(\xi, \tau, \delta)$. We then conclude the proof by iterating this method.

Arguing as above, we can easily prove the following property.

Remark 5.3. If the function $\mathcal{V}$ is bounded and $u$ is a solution to the problem

$$
\begin{cases}\mathcal{L}_{\mathcal{V}} u=f & \text { in } Q_{R}(\xi, \tau, T) \\ u=0 & \text { in } M_{R}(\xi, \tau, T) \\ u=g & \text { in } S_{R}(\xi, \tau)\end{cases}
$$

for some $f \in L^{1}\left(Q_{R}(\xi, \tau, T)\right)$ and $g \in C_{0}\left(S_{R}(\xi, \tau)\right)$, then

$$
u(z)=\int_{S_{R}(\xi, \tau)} G(z, y, \tau) g(y) d y-\int_{Q_{R}(\xi, \tau, T)} G(z, \eta) f(\eta) d \eta .
$$

In order to state our next result, we introduce some further notations. For a given $(\xi, \tau) \in \mathbb{R}^{N+1}$ and $R>0$, we set

$$
Q_{R}^{*}=Q_{R}^{*}\left(\xi, \tau, R^{2}\right)=Q_{R}\left(\xi^{*}, \tau^{*}, R^{2}\right), \quad \text { where } \quad\left(\xi^{*}, \tau^{*}\right)=(\xi, \tau) \circ\left(0,-R^{2}\right) .
$$

Note that $\tau^{*}=\tau-R^{2}$ by (2.4), then we may consider $Q_{R}^{*}$ as the cylinder whose upper basis is centered at $(\xi, \tau)$. We also set

$$
M(R)=\sup _{Q_{R}^{*}} u, \quad m(R)=\inf _{Q_{R}^{*}} u \quad \operatorname{Osc}(u, \xi, \tau, R)=M(R)-m(R) .
$$


Lemma 5.4. Let $u \geq 0$ be a bounded solution of $\mathcal{L}_{\mathcal{V}} u=0$ in $Q_{R}^{*}$. Then there exist $\delta, \varrho \in(0,1)$ and a positive $R_{0}$, which depend on $\eta_{\mathcal{V}}$ and $\mathcal{L}_{0}$, such that

$$
\operatorname{Osc}(u, \xi, \tau, \delta R) \leq \varrho M(R)
$$

for every $\left.R \in] 0, R_{0}\right]$.

Proof. The method is inspired by that in [29] (and has been used in [23]). Let $\varepsilon, \delta$ and $R_{0}$ be as in Proposition 4.5, and set

$$
\mathcal{S}=\left\{\left(x, \tau^{*}\right) \in S_{R}\left(\xi^{*}, \tau^{*}\right): u\left(x, \tau^{*}\right) \geq \frac{M(R)+m(R)}{2}\right\}
$$

Consider two possibilities.

Case 1: $\operatorname{meas}(\mathcal{S}) \geq \frac{1}{2} \operatorname{meas}\left(S_{R}\left(\xi^{*}, \tau^{*}\right)\right)$.

Define the function

$$
v(z)=\int_{S_{R}\left(\xi^{*}, \tau^{*}\right)} G_{0}\left(z, y, \tau^{*}\right)\left(u\left(y, \tau^{*}\right)-m(R)\right) d y+\int_{Q_{R}^{*}} G_{0}(z, \zeta) \mathcal{V}(\zeta) u(\zeta) d \zeta,
$$

and note that it is a solution to

$$
\begin{cases}\mathcal{L}_{0} v=-\mathcal{V} u & \text { in } Q_{R}\left(\xi^{*}, \tau^{*}, R^{2}\right) \\ v=u-m(R) & \text { in } S_{R}\left(\xi^{*}, \tau^{*}\right) \\ v \leq u-m(R) & \text { in } M_{R}\left(\xi^{*}, \tau^{*}, R^{2}\right)\end{cases}
$$

The function $u-m(R)$ is non-negative in $Q_{R}^{*}$ and $\mathcal{L}_{0}(u-m(R))=-\mathcal{V} u$, then, by the comparison principle, we find

$u(z)-m(R) \geq \int_{S_{R}\left(\xi^{*}, \tau^{*}\right)} G_{0}\left(z, y, \tau^{*}\right)\left(u\left(y, \tau^{*}\right)-m(R)\right) d y+\int_{Q_{R}^{*}} G_{0}(z, \zeta) \mathcal{V}(\zeta) u(\zeta) d \zeta$

for almost every $z \in Q_{R}^{*}$. We next apply Proposition 3.1 with $T=R^{2}$, and we obtain

$$
\begin{aligned}
& \int_{S_{R}\left(\xi^{*}, \tau^{*}\right)} G_{0}\left(z, y, \tau^{*}\right)\left(u\left(y, \tau^{*}\right)-m(R)\right) d y \geq \int_{\mathcal{S}} G_{0}\left(z, y, \tau^{*}\right)\left(u\left(y, \tau^{*}\right)-m(R)\right) d y \\
& \geq \frac{M(R)-m(R)}{2} \int_{\mathcal{S}} G_{0}\left(z, y, \tau^{*}\right) d y \geq \frac{M(R)-m(R)}{2} \int_{\mathcal{S}} \frac{\varepsilon}{\operatorname{meas}\left(S_{R}\left(\xi^{*}, \tau^{*}\right)\right)} d y \\
& \geq \frac{\varepsilon}{4}(M(R)-m(R)), \quad \text { for every } z \in Q_{\delta R}^{*}\left(\xi, \tau,(\delta R)^{2}\right) .
\end{aligned}
$$

On the other hand, we have

$$
\left|\int_{Q_{R}^{*}} G_{0}(z, \zeta) \mathcal{V}(\zeta) u(\zeta) d \zeta\right| \leq M(R) \eta_{\mathcal{V}}\left(R^{2}\right)
$$


where the integral is sufficiently small provided that we fix $R_{0}$ such that $\eta_{\mathcal{V}}\left(R^{2}\right) \leq \frac{\varepsilon}{8}$ for any $\left.\left.R \in\right] 0, R_{0}\right]$. Observing that

$$
m(\delta R)-m(R) \geq \frac{\varepsilon}{4}(M(R)-m(R))-\frac{\varepsilon}{8} M(R)
$$

it follows

$$
M(\delta R)-m(\delta R) \leq\left(1-\frac{\varepsilon}{4}\right)(M(R)-m(R))+\frac{\varepsilon}{8} M(R) \leq\left(1-\frac{\varepsilon}{8}\right) M(R) .
$$

This concludes the the proof in the first case, since $\varepsilon \in] 0,1[$.

Case 2: $\operatorname{meas}(\mathcal{S}) \leq \frac{1}{2} \operatorname{meas}\left(S_{R}\left(\xi^{*}, \tau^{*}\right)\right)$. In this case we set

$$
w(z)=\int_{S_{R}\left(\xi^{*}, \tau^{*}\right)} G_{0}\left(z, y, \tau^{*}\right)\left(M(R)-u\left(y, \tau^{*}\right)\right) d y+\int_{Q_{R}^{*}} G_{0}(z, \zeta) \mathcal{V}(\zeta) u(\zeta) d \zeta .
$$

Following the method used in Case 1 we find

$$
\begin{aligned}
M(R)-u(z) & \geq \int_{S_{R}\left(\xi^{*}, \tau^{*}\right) \backslash \mathcal{S}} G_{0}\left(z, y, \tau^{*}\right)\left(M(R)-u\left(y, \tau^{*}\right)\right) d y-\frac{\varepsilon}{8} M(R) \\
& \geq \frac{\varepsilon}{4}(M(R)-m(R))-\frac{\varepsilon}{8} M(R), \text { for a.e. } z \in Q_{\delta R}^{*}\left(\xi, \tau,(\delta R)^{2}\right)
\end{aligned}
$$

and then

$$
M(\delta R)-m(\delta R) \leq\left(1-\frac{\varepsilon}{8}\right) M(R) .
$$

The proof of Lemma 5.4 is then accomplished.

Proposition 5.5. Let $R_{0}$ and $\delta_{0}$ as in Proposition 4.5. Let $u \geq 0$ be a solution of $\mathcal{L}_{\mathcal{V}} u=0$ in $\Omega, Q_{R}\left(\xi, \tau, R^{2}\right) \subset \subset \Omega$, with $R \leq R_{0}$, and let $\mathcal{V}$ a bounded function. Then, for every $\alpha, \beta, \gamma, \delta \in] 0,1[$ such that $\alpha<\beta<\gamma$ and $\delta<\delta_{0}$ there exists a positive $M$ that depends on $\eta_{\mathcal{V}}, \eta_{\mathcal{V}}^{*}$ and on the constants $\alpha, \beta, \gamma, \delta$, but does not depend on the norm $\|\mathcal{V}\|_{L^{\infty}}$, such that

$$
\sup _{Q^{-}} u \leq M \inf _{Q^{+}} u \text {. }
$$

Proof. We first note that the boundedness of $\mathcal{V}$ yields the continuity of $u$, by the representation formula (3.10) and a standard bootstrap argument. Then there exists $(\bar{x}, \bar{t}) \in \overline{Q^{+}}$such that $u(\bar{x}, \bar{t})=\min \overline{Q^{+}} u$. It is not restrictive to assume $u(\bar{x}, \bar{t})=1$.

Following the line of the proof of Theorem 5.4 in [7], we consider, for every $r \in\left[0, \beta R^{2}\right]$, the following function

$$
v(x, t)=\int_{S_{R}(\xi, \tau, r)} G(x, t, y, r) u(y, r) d y, \quad \forall(x, t) \in Q_{R}\left(\xi, \tau, R^{2}\right) .
$$


By the comparison principle (recall that $\mathcal{V}$ is bounded and that $u \geq 0$ ) we obtain $u(x, t) \geq v(x, t)$, for every $(x, t) \in Q_{R}\left(\xi, \tau, R^{2}\right)$, then

$$
u(\bar{x}, \bar{t}) \geq \int_{S_{R}(\xi, \tau, r)} G(\bar{x}, \bar{t}, y, r) u(y, r) d y .
$$

Let us fix $\delta^{\prime}=\frac{\delta+\delta_{0}}{2}$ and consider, for any $\lambda>0$, the set

$$
\mathcal{S}(r, \lambda)=\left\{y \in S_{\delta^{\prime} R}(\xi, \tau, r): u(y, r) \geq \lambda\right\} .
$$

Then inequality (5.5) and Proposition 4.5 (with $\alpha_{0}=\gamma-\beta$ ) imply that

$$
1=u(\bar{x}, \bar{t}) \geq \int_{\mathcal{S}(r, \lambda)} G(\bar{x}, \bar{t}, y, r) u(y, r) d y \geq \frac{\lambda \varepsilon \operatorname{meas}(\mathcal{S}(r, \lambda))}{\operatorname{meas}\left(S_{R}(\xi, \tau)\right)} .
$$

We set

$$
K=\frac{1}{2}\left(1+\frac{1}{\varrho}\right) \quad r(\lambda)=\frac{R}{\delta}\left(\frac{4}{\varepsilon \lambda(1-\varrho)}\right)^{\frac{1}{Q-2}}
$$

where $\varrho$ is the constant in Lemma 5.4, and we note that

$$
Q_{\delta r(\lambda)}^{*}\left(\xi, \tau,(\delta r(\lambda))^{2}\right) \cap S_{R}(\xi, \tau, r)=S_{\delta r(\lambda)}(\zeta, \tau)
$$

for every $r \in\left[t-(\delta r(\lambda))^{2}, t\right]$. Then

$$
\operatorname{meas}\left(Q_{\delta r(\lambda)}^{*}\left(\xi, \tau,(\delta r(\lambda))^{2}\right) \cap S_{R}(\xi, \tau, r)\right)=\operatorname{meas}\left(S_{\delta r(\lambda)}(\zeta, \tau)\right)
$$

(by the analogous of (2.10) for the $N$-dimensional measure)

$$
=(\delta r(\lambda))^{Q-2} \cdot \operatorname{meas}(S)=\frac{4 R^{Q-2}}{\epsilon \lambda(1-\varrho)} \cdot \operatorname{meas}(S) .
$$

We next prove the following statement. Let $\lambda>0$ and $(x, t) \in Q_{\delta^{\prime} R}\left(\xi, \tau, R^{2}\right)$ with $t \leq \tau+\beta R^{2}$ be such that $u(x, t) \geq \lambda$ and that

$$
Q_{r(\lambda)}^{*}\left(x, t, r(\lambda)^{2}\right) \subset Q_{\delta^{\prime} R}\left(\xi, \tau, R^{2}\right) .
$$

Then there exists $\left(x^{\prime}, t^{\prime}\right) \in Q_{r(\lambda)}^{*}\left(x, t, r(\lambda)^{2}\right)$ such that $u\left(x^{\prime}, t^{\prime}\right) \geq K \lambda$.

Indeed, from (5.6) it follows that

$$
\operatorname{meas}\left(\mathcal{S}\left(r, \frac{\lambda}{2}(1-\varrho)\right)\right) \leq \frac{2 R^{Q-2}}{\lambda \varepsilon(1-\varrho)} \operatorname{meas}(S)
$$

so that, by (5.8), there is a $\left(\xi^{\prime}, \tau^{\prime}\right) \in Q_{\delta r(\lambda)}^{*}\left(\xi, \tau,(\delta r(\lambda))^{2}\right) \cap S_{R}(\xi, \tau, r)$ such that $u\left(\xi^{\prime}, \tau^{\prime}\right)<\frac{\lambda}{2}(1-\varrho)$. Our claim then follows from Lemma 5.4. 
We next show that there exists a positive constant $M$ such that $u(x, t)$ $\leq M$ for every $(x, t) \in Q^{-}$. The thesis then follows, since $u(\bar{x}, \bar{t})=\min \overline{Q^{+}} u=1$. Suppose, by contradiction, that there were a $z_{0} \in Q^{-}$such that $u\left(z_{0}\right)>M$. Then, repeating the arguments used above to obtain $u\left(x^{\prime}, t^{\prime}\right) \geq K \lambda$, there exists a sequence $\left(z_{j}\right)$ such that

$$
u\left(z_{j}\right) \geq M K^{j}, \quad z_{j+1} \in Q_{r_{j}}^{*}\left(z_{j}, r_{j}^{2}\right), \quad \text { where } r_{j}=r\left(M K^{j}\right),
$$

provided that

$$
Q_{r_{j}}^{*}\left(z_{j}, r_{j}^{2}\right) \subset Q_{\delta^{\prime} R}\left(\xi, \tau, R^{2}\right), \quad \text { for every } j \in \mathbb{N} .
$$

In order to prove (5.9) we note that

$$
d\left(z_{j+1}, z_{j}\right) \leq c_{0} r_{j}=c_{0} \frac{R}{\delta}\left(\frac{4}{\varepsilon M(1-\varrho) K^{j}}\right)^{\frac{1}{Q-2}}
$$

where $c_{0}=\max _{z \in Q_{1}^{*}(0,0,1)} d(z,(0,0))$ (recall $\left.(2.1)\right)$. Hence

$$
d\left(z_{j}, z_{0}\right) \leq c_{0} \frac{R}{\delta}\left(\frac{4}{\varepsilon M(1-\varrho) K^{j}}\right)^{\frac{1}{Q-2}} \sum_{i=1}^{\infty} K^{-\frac{i}{Q-2}},
$$

so that we can choose a positive $M$, that depends on $\alpha, \delta, \delta_{0}$ but does not depend on $R$, such that (5.9) holds. Hence the sequence $u\left(z_{j}\right)$ is unbounded and we get a contradiction with the continuity of $u$. This accomplishes the proof.

In a similar way it is true the next result for the adjoint operator.

Remark 5.6. Let $v \geq 0$ be a solution of $\mathcal{L}_{\mathcal{V}}^{*} v=0$ in $Q_{R}\left(\xi, \tau, R^{2}\right)$, where $\mathcal{V}$ is a bounded function and it is in the class $S K\left(Q_{R}\left(\xi, \tau, R^{2}\right), \mathcal{L}_{0}\right)$. Then

$$
\sup _{Q^{+}} v \leq M \inf _{Q^{-}} v
$$

for some positive constant $M$ depending on $\eta_{\mathcal{V}}, \eta_{\mathcal{V}}^{*}$ and on the constants $\alpha, \beta, \gamma, \delta$, but that does not depend on $\|\mathcal{V}\|_{L^{\infty}}$.

Lemma 5.7. Let $u$ be a solution of $\mathcal{L}_{\mathcal{V}} u=0$ in $\Omega$. Then, for any $z_{0} \in \Omega$ there exists a compact neighborhood $K$ of $z_{0}$ such that $K \subset \Omega$ and that $u$ is the limit in $L^{1}(K)$ of a sequence $\left(u_{m}\right)_{m \in \mathbb{N}}$, where every $u_{m}$ satisfies

$$
\mathcal{L}_{\mathcal{V}_{m}} u_{m}=0 \text { in } K \text {. }
$$

Moreover, for every compact set $H \subset \operatorname{int}(K)$, there exists a positive constant $c_{H}$ such that

$$
\left|u_{m}(z)\right| \leq c_{H} \quad \forall z \in H, \quad \forall m \in \mathbb{N} .
$$


Proof . Consider a cylindrical set $Q_{R}(\xi, \tau, T)$ such that $\overline{Q_{R}(\xi, \tau, T)} \subset \Omega$, and suppose that $\eta_{\mathcal{V}}(T)<1$ and $\eta_{\mathcal{V}}^{*}(T)<1$. Let also consider a text function $\varphi \in C_{0}^{\infty}\left(Q_{R}(\xi, \tau, T)\right)$ such that $\varphi \equiv 1$ in a compact neighborhood $K$ of $z_{0}$ such that $K \subset Q_{R}(\xi, \tau, T)$. We have

$$
\mathcal{L}_{\mathcal{V}_{m}}(u \varphi)=\varphi \mathcal{L}_{0} u+u \mathcal{L}_{0} \varphi+2 \sum_{j=1}^{m} X_{j} \varphi X_{j} u+u \varphi \mathcal{V}_{m}=
$$

(recalling that $u$ is solution of $\mathcal{L}_{0} u+\mathcal{V} u=0$ )

$$
=u \mathcal{L}_{0} \varphi+2 \sum_{j=1}^{m} X_{j} \varphi X_{j} u+\left(\mathcal{V}_{m}-\mathcal{V}\right) u \varphi
$$

In the sequel we will set $f=2 \sum_{j=1}^{m} X_{j} \varphi X_{j} u+u \mathcal{L}_{0} \varphi$. We also consider the Green function $G_{m}$ related to $\mathcal{L}_{\mathcal{V}_{m}}$ and set

$$
u_{m}(z)=-\int_{Q_{R}(\xi, \tau, T)} G_{m}(z, \zeta) f(\zeta) d \zeta .
$$

We have

$$
\begin{cases}\mathcal{L}_{m}\left(u_{m}-\varphi u\right)=-\left(\mathcal{V}_{m}-\mathcal{V}\right) \varphi u & \text { in } Q_{R}(\xi, \tau, T) \\ u_{m}-\varphi u=0 & \text { in } \partial_{r} Q_{R}(\xi, \tau, T)\end{cases}
$$

and the function $\left(\mathcal{V}_{m}-\mathcal{V}\right) \varphi u$ belongs to $L^{1}\left(Q_{R}(\xi, \tau, T)\right)$, then, by Remark 5.3, we find

$$
\left(u_{m}-\varphi u\right)(z)=\int_{Q_{R}(\xi, \tau, T)} G_{m}(z, \zeta)\left(\mathcal{V}_{m}-\mathcal{V}\right) \varphi u(\zeta) d \zeta
$$

We next integrate over $Q_{R}(\xi, \tau, T)$ and use property (i) of Proposition 4.2, for $p=1$. We obtain

$$
\left\|u_{m}-\varphi u\right\|_{L^{1}\left(Q_{R}(\xi, \tau, T)\right)} \leq c_{1}\left\|\left(\mathcal{V}_{m}-\mathcal{V}\right) \varphi u\right\|_{L^{1}\left(Q_{R}(\xi, \tau, T)\right)},
$$

for some constant $c_{1}$ that does not depend on $m$. On the other hand

$$
\left|\left(\mathcal{V}_{m}(\zeta)-\mathcal{V}(\zeta)\right) \varphi(\zeta) u(\zeta)\right| \leq|\mathcal{V}(\zeta) \varphi(\zeta) u(\zeta)|,
$$

for almost every $\zeta \in Q_{R}(\xi, \tau, T)$ and the function $\mathcal{V} \varphi u \in L^{1}\left(Q_{R}(\xi, \tau, T)\right)$, then

$$
\lim _{m \rightarrow \infty}\left\|u_{m}-\varphi u\right\|_{L^{1}\left(Q_{R}(\xi, \tau, T)\right)}=0 .
$$

This proves the first claim.

We next prove (5.10). We set

$$
\widetilde{H}=\operatorname{supp}\left(\left(X_{1} \varphi\right)^{2}+\cdots+\left(X_{m} \varphi\right)^{2}+(Y \varphi)^{2}\right)
$$

and note that $f(\zeta)=0$ for every $\zeta \notin \widetilde{H}$. 
We next prove that there exists a positive constant $\widetilde{c}$, that depends on $H$ and $\widetilde{H}$, but does not depends on $m$, such that

$$
G_{m}(z, \zeta) \leq \widetilde{c} \text { for every } z \in H, \zeta \in \widetilde{H} .
$$

As a consequence, by formula (5.11) we obtain

$$
\left|u_{m}(z)\right| \leq \int_{\widetilde{H}} G_{m}(z, \zeta) f(\zeta) d \zeta \leq \widetilde{c}\|f\|_{L^{1}\left(Q_{R}(\xi, \tau, T)\right)}
$$

for every $z \in H$, and the proof is concluded, since

$$
\|f\|_{L^{1}\left(Q_{R}(\xi, \tau, T)\right)} \leq k\left(\sum_{j=1}^{m}\left\|X_{j} u\right\|_{L^{1}\left(Q_{R}(\xi, \tau, T)\right)}+\|u\|_{L^{1}\left(Q_{R}(\xi, \tau, T)\right)}\right),
$$

for a positive constant $k$ that only depends on $\varphi$ and on $\mathcal{L}_{0}$.

We prove (5.12) by using the Harnack inequality stated in Remark 5.6. Let $z$ be a point of $H$. For every $\zeta \in \widetilde{H}$ we consider a cylindrical open set $\widetilde{Q} \subset Q_{R}(\xi, \tau, T)$ such that $\zeta \in \widetilde{Q}^{+}$and $\widetilde{Q} \cap H=\emptyset$. Since $G_{m}(z, \cdot)$ is a positive solution to $\mathcal{L}_{\mathcal{V}_{m}}^{*} v=0$, by Remark 5.6 we have

$$
\sup _{\widetilde{Q}^{+}} G_{m}(z, \cdot) \leq M \inf _{\widetilde{Q}^{-}} G_{m}(z, \cdot)
$$

for some positive constant $M$ that does not depend on $m$. On the other hand

$$
\operatorname{meas}\left(\widetilde{Q}^{-}\right) \inf _{\widetilde{Q}^{-}} G_{m}(z, \cdot) \leq \int_{Q_{R}(\xi, \tau, T)} G_{m}(z, \zeta) d \zeta \leq c_{1} \sum_{k=0}^{\infty} \eta_{\mathcal{V}}(T)^{k},
$$

where $c_{1}$ is the constant appearing in the statement (i) of Proposition 4.2. Thus $G_{m}(z, \zeta) \leq \widetilde{k}$, for every $z \in H$ and $\zeta \in \widetilde{Q}^{+}$, where the constant $\widetilde{k}$ depends on $M, c_{1}$ and $\eta_{\mathcal{V}}(T)$. The estimate (5.12) then follows from a standard covering argument for the compact set $\widetilde{H}$. This completes the proof.

Proof of Proposition 2.3. Let $u$ be a solution of the equation $\mathcal{L}_{\mathcal{V}} u=0$ in $\Omega$. By Lemma 5.7, $u$ is the limit, in $L_{\text {loc }}^{1}(\Omega)$ of a sequence of bounded functions $\left(u_{m}\right)_{m \in \mathbb{N}}$ such that $\mathcal{L}_{\mathcal{V}_{m}} u_{m}=0$ in a suitable compact set $K \subset \Omega$. We then apply Lemma 5.1 to every function $u_{m}$, then there exists a subsequence $\left(u_{m_{k}}\right)_{k \in \mathbb{N}}$ that converges uniformly to $u$ in $K$. Thus the estimate of the modulus of continuity stated in Lemma 5.1 extends to $u$. This completes the proof. 
Proof of Theorem 2.1. Let $Q_{R}\left(\xi_{0}, \tau_{0}, T\right)$ be any cylindrical set. If $\eta_{V}(T)$ $<1$ and $\eta_{V}^{*}(T)<1$, the result immediately follows from Proposition 4.2.

If otherwise $\eta_{V}(T) \geq 1$, or $\eta_{V}^{*}(T) \geq 1$, we choose $h>0$ such that $\eta_{V}(h)<1, \eta_{V}^{*}(h)<1$. Consider the cylinders

$$
\begin{array}{ll}
\left.Q^{(s)}\left(T_{0}\right)=O \times\right] s, s+T_{0}[, & Q_{R}^{(s)}\left(\xi_{0}, \tau_{0}, T_{0}\right)=\left(\xi_{0}, \tau_{0}\right) \circ \delta_{R} Q^{(s)}\left(R^{-2} T_{0}\right) \\
S^{(s)}=O \times\{s\}, & S_{R}^{(s)}\left(\xi_{0}, \tau_{0}\right)=\left(\xi_{0}, \tau_{0}\right) \circ \delta_{R} S^{(s)},
\end{array}
$$

and let $G^{(s)}$ denote the Green function related to $Q_{R}^{(s)}\left(\xi_{0}, \tau_{0}, h\right)$ (we can employ the argument used in Proposition 4.2 without any change). We then extend the definition of $G$ given in Proposition 4.2 as follows: for every $(x, t) \in Q_{R}\left(\xi_{0}, \tau_{0}, T\right)$ such that $s+h<t \leq s+2 h$, we set

$$
G(x, t, y, s)=\int_{S_{R}^{(s)}\left(\xi_{0}, \tau_{0}\right)} G^{(s+h)}(x, t, w, s+h) G^{(s)}(w, s+h, y, s) d w .
$$

It is easy to verify that $G$ is a Green function for the set $Q_{R}\left(\xi_{0}, \tau_{0}, 2 h\right)$ and that $G^{*}(\zeta, z)=G(z, \zeta)$ is a Green function for the adjoint operator $\mathcal{L}_{\mathcal{V}}{ }^{*}$. For $(x, t) \in Q_{R}\left(\xi_{0}, \tau_{0}, T\right)$ such that $s+2 h<t \leq s+4 h$ we repeat the above argument and define the Green function in the set $Q_{R}\left(\xi_{0}, \tau_{0}, 4 h\right)$ as

$$
G(x, t, y, s)=\int_{S_{R}^{(s)}\left(\xi_{0}, \tau_{0}\right)} G^{(s+2 h)}(x, t, w, s+2 h) G^{(s)}(w, s+2 h, y, s) d w .
$$

After a finite number of iterations we obtain a Green function for the $Q_{R}\left(\xi_{0}, \tau_{0}, T\right)$. This completes the proof.

Proof of Theorem 2.2. As in Proposition 2.3, we obtain the result by using Lemma 5.7 and Proposition 5.5.

Acknowledgments. We wish to thank E. Lanconelli for his interest in this problem and for many helpful discussions.

\section{References}

[1] Alexopoulos, G. K.: Sub-Laplacians with drift on Lie groups of polynomial volume growth. Mem. Amer. Math. Soc. 155, no. 739, (2002).

[2] Bonfiglioli, A., Lanconelli, E. And Uguzzoni, F.: Stratified Lie groups and potential theory for their sub-Laplacians. Springer Monographs in Mathematics. Springer, Berlin, 2007.

[3] Bony, J. M.: Principe du maximum, inégalite de Harnack et unicité du problème de Cauchy pour les opérateurs elliptiques dégénérés. Ann. Inst. Fourier (Grenoble) 19 (1969), 277-304.

[4] Bramanti, M. and Brandolini, L.: Estimates of BMO type for singular integrals on spaces of homogeneous type and applications to hypoelliptic PDEs. Rev. Mat. Iberoamericana 21 (2005), no. 2, 511-556. 
[5] Citti, G., Garofalo, N. and Lanconelli, E.: Harnack's inequality for sum of squares of vector fields plus a potential. Amer. J. Math. 115 (1993), no. 3, 699-734.

[6] Fabes, E. B. and Stroock, D. W.: The $L^{p}$-integrability of Green's functions and fundamental solutions for elliptic and parabolic equations. Duke Math. J. 51 (1984), no. 4, 997-1016.

[7] Fabes, E. B. And Stroock, D. W.: A new proof of Moser's parabolic Harnack inequality using the old ideas of Nash. Arch. Rational Mech. Anal. 96 (1986), no. 4, 327-338.

[8] Folland, G. B.: Subelliptic estimates and function spaces on nilpotent Lie groups. Ark. Mat. 13 (1975), no. 2, 161-207.

[9] Garofalo, N. and Lanconelli, E.: Level sets of the fundamental solution and Harnack inequality for degenerate equations of Kolmogorov type. Trans. Amer. Math. Soc. 321 (1990), no. 2, 775-792.

[10] Hörmander, L.: Hypoelliptic second order differential equations. Acta Math. 119 (1967), 147-171.

[11] KogoJ, A. And Lanconelli, E.: An invariant Harnack inequality for a class of hypoelliptic ultraparabolic equations. Med. J. Math. 1 (2004), no. $1,51-80$.

[12] KogoJ, A. And Lanconelli, E.: One-side Liouville theorems for a class of hypoelliptic ultraparabolic equations. In Geometric Analysis of PDE and Several Complex Variables, 305-312. Contemp. Math. 368. Amer. Math. Soc., Providence, RI, 2005.

[13] KogoJ, A. And Lanconelli, E.: Link of groups and homogeneous Hörmander operators. Proc. Amer. Math. Soc. 135 (2007), no. 7, 2019-2030.

[14] Kuptsov, L. P.: On parabolic means. Dokl. Acad. Nauk SSSR 252 (1980), no. 2, 296-301.

[15] Kusuoka, S. And Stroock, D. W.: Applications of the Malliavin calculus, III. J. Fac. Sci. Univ. Tokyo Sect. IA Math. 34 (1987), no. 2, 391-442.

[16] Lanconelli, E., Pascucci, A. and Polidoro, S.: Linear and nonlinear ultraparabolic equations of Kolmogorov type arising in diffusion theory and in finance. In Nonlinear problems in mathematical physics and related topics, II, 243-265. Int. Math. Ser. 2. Kluwer/Plenum, New York, 2002.

[17] Lanconelli, E. And Pascucci, A.: On the fundamental solution for hypoelliptic second order partial differential equations with non-negative characteristic form. Ricerche Mat. 48 (1999), no. 1, 81-106.

[18] Lanconelli, E. And Polidoro, S.: On a class of hypoelliptic evolution operators. Rend. Sem. Mat. Univ. Politec. Torino 52 (1994), no. 1, 29-63.

[19] LU, G.: On Harnack's inequality for a class of strongly degenerate Schrödinger operators formed by vector fields. Differential Integral Equations $\mathbf{7}$ (1994), no. 1, 73-100.

[20] Montanari, A.: Harnack inequality for totally degenerate KolmogorovFokker-Planck operators. Boll. Un. Mat. Ital. (7) 10 (1996), no. 4, 903-926. 
[21] Nagel, A., Stein, E. M. And Wainger, S.: Balls and metrics defined by vector fields. I. Basic properties. Acta Math. 155 (1985), no. 1-2, 103-147.

[22] Pascucci, A. And Polidoro, S.: Harnack inequalities and Gaussian estimates for a class of hypoelliptic operators. Trans. Amer. Math. Soc. 358 (2006), no. 11, 4873-4893.

[23] Polidoro, S. And Ragusa, M. A.: A Green function and regularity results for an ultraparabolic equation with a singular potential. Adv. Differential Equations 7 (2002), no. 11, 1281-1314.

[24] Rothschild, L. P. And Stein, E. M.: Hypoelliptic differential operators and nilpotent groups. Acta Math. 137 (1977), no. 3-4, 247-320.

[25] Safanov, M. N.: Harnack's inequality for elliptic equations and the Hölder property of their solutions. J. Soviet Mathematics 21 (1983), 851-863.

[26] Schechter, M.: Spectra of partial differential operators. North-Holland Series in Applied Mathematics and Mechanics 14. North-Holland Publishing Co., Amsterdam, 1971.

[27] Sturm, K. T.: Harnack's inequality for parabolic operators with singular low order terms. Math. Z. 216 (1994), no. 4, 593-611.

[28] Varopoulos, N. Th., Saloff-Coste, L. and Coulhon, T.: Analysis and geometry on groups Cambridge Tracts in Mathematics 100. Cambridge University Press, Cambridge, 1992.

[29] Zhang, Q.: On a parabolic equation with a singular lower order term. Trans. Amer. Math. Soc. 348 (1996), no. 7, 2811-2844.

[30] ZHANG, Q.: On a parabolic equation with a singular lower order term, II. The Gaussian bounds. Indiana Univ. Math. J. 46 (1997), no. 3, 989-1020.

Recibido: 15 de julio de 2007

Revisado: 8 de octubre de 2007

Sergio Polidoro

Dipartimento di Matematica Pura e Applicata Università degli Studi di Modena e Reggio Emilia

Via G. Campi 213/b

41100 Modena-Italy

sergio.polidoro@unimore.it

Maria Alessandra Ragusa

Dipartimento di Matematica

Università di Catania

Viale Andrea Doria, 6

95125 Catania, Italy

maragusa@dmi.unict.it 